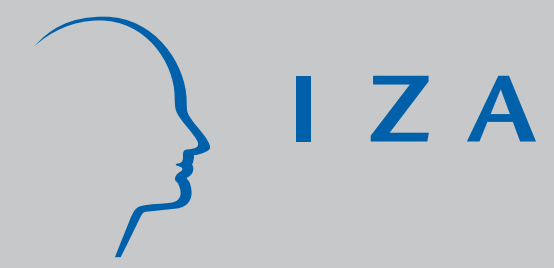

IZA DP No. 997

J ob Search and Hyperbolic Discounting:

Structural Estimation and Policy Evaluation

M. Daniele Paserman

J anuary 2004 


\title{
Job Search and Hyperbolic Discounting: Structural Estimation and Policy Evaluation
}

\author{
M. Daniele Paserman \\ Hebrew University and IZA Bonn \\ Discussion Paper No. 997 \\ January 2004
}

\author{
IZA \\ P.O. Box 7240 \\ D-53072 Bonn \\ Germany \\ Tel.: +49-228-3894-0 \\ Fax: +49-228-3894-210 \\ Email: iza@iza.org
}

\begin{abstract}
This Discussion Paper is issued within the framework of IZA's research area Evaluation of Labor Market Policies and Projects. Any opinions expressed here are those of the author(s) and not those of the institute. Research disseminated by IZA may include views on policy, but the institute itself takes no institutional policy positions.
\end{abstract}

The Institute for the Study of Labor (IZA) in Bonn is a local and virtual international research center and a place of communication between science, politics and business. IZA is an independent, nonprofit limited liability company (Gesellschaft mit beschränkter Haftung) supported by Deutsche Post World Net. The center is associated with the University of Bonn and offers a stimulating research environment through its research networks, research support, and visitors and doctoral programs. IZA engages in (i) original and internationally competitive research in all fields of labor economics, (ii) development of policy concepts, and (iii) dissemination of research results and concepts to the interested public. The current research program deals with (1) mobility and flexibility of labor, (2) internationalization of labor markets, (3) welfare state and labor market, (4) labor markets in transition countries, (5) the future of labor, (6) evaluation of labor market policies and projects and (7) general labor economics.

IZA Discussion Papers often represent preliminary work and are circulated to encourage discussion. Citation of such a paper should account for its provisional character. A revised version may be available on the IZA website (www.iza.org) or directly from the author. 


\section{ABSTRACT \\ Job Search and Hyperbolic Discounting:
Structural Estimation and Policy Evaluation*}

This paper estimates the structural parameters of a job search model with hyperbolic discounting and endogenous search effort. It estimates quantitatively the degree of hyperbolic discounting, and assesses its implications for the impact of various policy interventions aimed at reducing unemployment. The model is estimated using data on unemployment spells and accepted wages from the National Longitudinal Survey of Youth (NLSY). The likelihood function explicitly incorporates all the restrictions implied by the optimal dynamic programming solution to the model. Both observed and unobserved heterogeneity are accounted for. The results point to a substantial degree of hyperbolic discounting, especially for low and medium wage workers. The structural estimates are also used to evaluate alternative policy interventions for the unemployed. Estimates based on a model with exponential discounting may lead to biased inference on the economic impact of policies.

JEL Classification: C11, C41

Keywords: job search, hyperbolic discounting, structural estimation, policy evaluation

M. Daniele Paserman

Department of Economics

Hebrew University

Jerusalem, 91905

Israel

Email: dpaserma@shum.huji.ac.il

\footnotetext{
* I thank Gary Chamberlain, Stefano DellaVigna, Eric Gould, David Laibson, Lawrence Katz, Robert Sauer and participants in seminars at the Bank of Israel, Bar Ilan University, Ben Gurion University, Ente Luigi Einaudi, Harvard University, the Hebrew University, Univeristy of Toulouse, the Econometrics in Tel Aviv 2001 conference, and the 2002 Econometric Society Winter Meetings for many helpful comments. Michael Hvoshniansky provided helpful research assistance. Financial support from the Maurice Falk Institute for Economic Research in Israel and from the Israeli Science Foundation is gratefully acknowledged. All errors are my own.
} 


\section{Introduction}

Job search is an unpleasant activity with immediate costs and delayed benefits. The tension between long-run goals and short-run impulses may lead unemployed workers to postpone repeatedly tasks necessary to find a job. In standard economic models, agents are assumed to be time-consistent, so that a contrast between short-run and long-run preferences never arises. However, a growing literature has challenged the conventional view, and allows agents to be time inconsistent by modeling their discount function as hyperbolic (as opposed to the standard assumption of exponential discounting). ${ }^{1}$ Agents with hyperbolic discount functions exhibit a high degree of discounting in the short run, but a relatively low degree of discounting in the long run. Therefore, hyperbolic agents are likely to delay tasks with immediate costs and delayed benefits, whereas they would choose to perform the same task if both costs and benefits were to occur in the future.

Hyperbolic preferences may affect the way we think of job search problems, and of policies addressing unemployment. First, we must recognize that agents with hyperbolic and exponential preferences will give different weights to the various components of costs and benefits involved in the search process: hyperbolic agents will be particularly sensitive to the immediate and direct costs of search (writing the résumé, contacting employers, making unpleasant phone calls to distant relatives), while impatient exponential agents are more likely to be affected by long-run costs and benefits, such as those associated with waiting longer to obtain a better job. Hence, policies that are targeted at one particular dimension of the job search process may be more effective for one type of worker than for the other. Secondly, the welfare implications of unemployment policies may differ substantially depending on the type of preferences. In a model with conventional preferences, an agent may experience a long unemployment spell because of bad luck. With hyperbolic preferences, a long unemployment spell could be due to "bad" choices, with the agent agreeing that his own choices are undesirable from a longrun perspective. Therefore, an intervention that brings the agent to choose actions more in line with his long-run preferences may actually be welfare improving, despite its imposition of restrictions, potentially even highly unpleasant ones, on the unemployed.

In order to assess the effects of policies, we need to estimate the degree of hyperbolic discounting. Despite the recent uspurge of interest in time-inconsistent preferences, direct estimates of the parameters of the discount function are relatively rare. ${ }^{2}$ Knowledge of these parameters has important implications not only for the specific environment studied in this paper (job search

\footnotetext{
${ }^{1}$ See Laibson (1997); O’Donoghue and Rabin (1999); Harris and Laibson (2001).

${ }^{2}$ Exceptions include Angeletos et al. (2001), and Fang and Silverman (2002).
} 
and unemployment spells), but also for the multitude of other applications in which hyperbolic preferences have been used, from retirement savings (Laibson, Repetto and Tobacman, 1998 and 2003; Diamond and Köszegi, 2003) to the consumption of addictive goods (Gruber and Köszegi, 2001).

In this paper, I provide one of the first structural estimates of the degree of hyperbolic discounting. I set up a model of job search with endogenous search effort and hyperbolic discounting, similar to the one used in DellaVigna and Paserman (2000 henceforth DV-P). I set up a likelihood function that explicitly incorporates all the restrictions implied by the optimal dynamic programming solution to the model. The model is estimated using data on unemployment spells and accepted wages from the National Longitudinal Survey of Youth (NLSY). I control for observed heterogeneity in the worker's wages before the unemployment spell, in cognitive test scores, in marital status, and in race. Unobserved heterogeneity enters the model as a finite mixture distribution, whose parameters need to be estimated.

A key question that arises in empirical studies on hyperbolic preferences is that of identification. In many settings, the behavior of agents with hyperbolic preferences is observationally equivalent to that of agents with conventional preferences but a high degree of impatience. The distinguishing feature of the model presented here is that different forms of impatience have contrasting effects on the outcomes of the job search process. In DV-P we showed that impatience lowers the probability of receiving a job offer, but raises the probability of accepting the offer. For hyperbolic workers, the first effect dominates, so that, ceteris paribus, workers with a higher degree of short-run impatience experience longer spells of unemployment. On the other hand, if individuals only differ in their exponential discount rate, the second effect in general dominates: more impatient exponential workers have shorter unemployment spells. The intuition behind the main result in DV-P helps to illustrate how the short-run and the longrun discounting parameters are separately identified with data on the length of unemployment spells and accepted wages. The search process is made up of two separate decisions: the search effort decision involves a trade-off between immediate costs and benefits that will be realized in the near future, once an offer is accepted. In the United States, this time span is typically no longer than 30 weeks. Over this limited time horizon, short-run impatience matters the most. The reservation wage decision, on the other hand, essentially involves only a comparison between payoffs in the more distant future. To a first approximation, the degree of short-run discounting has no effect on the reservation wage. ${ }^{3}$ Then, assuming that all the other model

\footnotetext{
${ }^{3}$ The statement is exactly true if agents are naive and there is a one period delay between the time a job offer is accepted and the time of the first wage payment. See DV-P, Proposition 1(c).
} 
parameters are known, one can back out the long-term discount factor from the estimated reservation wage. The short term discount factor is then identified from data on exit rates from unemployment.

The results of the structural estimation point to a substantial degree of present bias for low and medium wage workers, and only a moderate degree of short run impatience for high wage workers. I use the maximum likelihood estimates to evaluate alternative policy interventions for the unemployed. The policies analyzed are a cut in unemployment benefits, a job search assistance program, monitoring search effort, monitoring the job acceptance strategy, and a re-employment bonus. I find that ignoring hyperbolic preferences may lead one to incorrect inferences on the effects of these interventions.

The rest of the paper is organized as follows. In Section 2, after a brief summary of hyperbolic discounting, I describe the model and the main features of its solution. In Section 3 I describe how to estimate the structural parameters of the model. I set up the likelihood function, specify functional forms, and discuss identification issues. In Section 4 I present and discuss the results of the maximum likelihood estimation. I also analyze the robustness of the results to different specifications of the heterogeneity distribution, and investigate the predictions generated by the different specifications with respect to several outcomes of interest, both in terms of the outcomes' expected values ex-ante, and in terms of their dynamic evolution over the course of the unemployment spell. In Section 5, I present the results of my simulated policy evaluations. Section 6 concludes.

\section{The Model}

\subsection{Hyperbolic discounting}

Over the years, psychologists have collected a substantial body of evidence on individual time preferences (for a review, see Ainslie, 1992). Experiments show that agents are extremely impatient if the rewards are to be obtained in the near future, but relatively patient when choosing between rewards to be accrued in the distant future. This form of discounting implies that agents prefer a larger, later reward over a smaller, earlier one as long as the rewards are sufficiently distant in time; however, as both rewards get closer in time, the agent may choose the smaller, earlier reward. In an experiment with monetary rewards an overwhelming majority of subjects exhibit such reversal of preferences (Kirby and Herrnstein, 1995).

Hyperbolic discount functions, introduced by Phelps and Pollak (1968) and first studied in 
the context of intertemporal one-person decisions by Laibson (1997), provide a convenient representation of the above findings: for a decision maker at any time $s$, the discount function is equal to 1 for $t=s$ and to $\beta \delta^{t-s}$ for $t=s+1, s+2, \ldots$, with $\beta<1$. The implied discount factor from period $s$ to the next period is $\beta \delta$, while the discount factor between any two periods in the future is simply $\delta$. However, when period $s+1$ comes along, the agent faces the same type of preferences: the discount factor between that period and the next is $\beta \delta$. This matches the main feature of the experimental evidence - high discounting at short delays, low discounting at long delays.

These preferences are dynamically inconsistent. To illustrate this, consider a plan of actions $q_{t}$ that yield instantaneous utility $u\left(q_{t}\right)$, for $t=0,1,2, \ldots, T$. From today's perspective, the plan from period $s$ onwards yields utility

$$
V^{s}\left(q_{s}, \ldots, q_{T}\right)=\beta \sum_{t=s}^{T} \delta^{t} u\left(q_{t}\right) .
$$

However, from the perspective of the decision maker at time $s$, the same plan yields utility

$$
\tilde{V}^{s}\left(q_{s}, \ldots, q_{T}\right)=u\left(q_{s}\right)+\beta \sum_{t=s+1}^{T} \delta^{t-s} u\left(q_{t}\right) .
$$

There is therefore a conflict between the preferences of a given individual at different points in time. Note that this conflict disappears in the special case of $\beta=1$. In this case, we are back to the time-consistent exponential model with discount function $\delta^{t}$.

We can interpret $\beta$ as the parameter of short-run patience and $\delta$ as the parameter of long-run patience. The degree of short-run patience $\beta$ is crucial for this theory; any $\beta$ smaller than one is sufficient to generate some degree of procrastination in activities with salient costs and delayed benefits, such as searching for a job (O'Donoghue and Rabin, 1999). The implications of this form of time inconsistency in the context of job search are easy to see. A worker with a very high degree of short run impatience may wish to postpone her job search activities to a later period, when, from today's perspective, the discount rate is relatively low; however, when the later period comes along, the worker once more faces a high degree of short-run impatience, and will choose to postpone her activity again.

For the purposes of this paper, we will restrict attention to the case of a sophisticated hyperbolic worker. A sophisticated agent is aware of her time inconsistency problem: she knows that at time $s$ in the future, she will choose an action $q_{s}^{*}$ that maximizes the utility function in $(2.2)$. The optimal choice at $s$ depends on the set of actions chosen from today until $s-1$. Therefore 
in the present she chooses $q_{0}$ that solves:

$$
\max _{q_{0}} u\left(q_{0}\right)+\beta \sum_{t=1}^{T} \delta^{t} u\left[q_{t}^{*}\left(q_{0}\right)\right] .
$$

The sophisticated agent knows that if she postpones a task until tomorrow, she may wish to postpone it again. She will therefore try to find ways to overcome her procrastination problems. In the context of job search, the sophisticated worker will, assuming that no commitment devices are available, exert higher effort in the present, knowing that in the future she will search less than what is optimal from today's perspective. ${ }^{4}$

\subsection{Setting}

The model is a variant of the prototypical job search model (Lippman and McCall, 1976), augmented to include endogenous search effort and hyperbolic discounting. The model is set in discrete time; it is convenient to think of a week as the time unit. Consider an infinitely lived worker who is unemployed at time $t$. In each period of unemployment, the worker takes two decisions: first, he chooses the amount of search effort; second, conditional on receiving a job offer, he decides whether to accept it or not.

In every period, the worker receives instantaneous utility $b_{t}$. I model $b_{t}$ as the sum of two components: the first component, $b_{t}^{U I}$ is the monetary value of Unemployment Insurance (UI) benefits. This may vary during the course of the unemployment spell. The second component, $b_{0}$, represents the psychic value of being unemployed: it can be either positive (utility of leisure) or negative (stigma, low self-esteem, etc.). I assume that $b_{0}$ is invariant over time.

Search effort $s_{t}$ is parameterized as the probability of obtaining a job offer; ${ }^{5}$ therefore, $s_{t} \in[0,1]$. In every period the agent incurs a cost of search $c\left(s_{t}\right)$, an increasing and strictly convex function of $s_{t}$. The assumption of increasing marginal cost comes naturally. The marginal cost of effort is low when the agent is searching little: a meeting with a friend or a glance at the newspaper raises the probability of finding a job at minimal additional expense. On the other hand, increasing the probability of receiving an offer up to almost certainty is likely to have

\footnotetext{
${ }^{4} \mathrm{~A}$ naive worker believes incorrectly that her future preferences will be exponential, and that his procrastination problems are only temporary. In DV-P, we found that the qualitative and quantitative behavior of sophisticated and naive workers in a job search model is similar.

${ }^{5}$ Suppose that the worker chooses search effort $z$, and that a job offer is generated with probability $s=p(z)$. Under the assumption that $p(\cdot)$ is a strictly increasing function of search, there is a one-to-one correspondence between $s$ and $z$. Without loss of generality, we can then specify the worker's problem more conveniently in terms of the probability of receiving an offer $s$.
} 
prohibitive costs. In order to simplify the characterization of the solution, I also assume no fixed costs of search: a worker may be informed about an outstanding job offer at no cost.

Upon receiving a job offer, the worker must decide whether to accept it or not. The job offer is characterized by a wage $w$, which is a realization of a random variable $W$ with cumulative distribution function $F$. If the worker accepts the offer, he becomes employed and receives wage $w$ starting from next period. We assume $F$ to be known to the worker, constant over time and independent of search effort. In other words, search effort determines how often the individual samples out of $F$, not the distribution being sampled.

Finally, we allow for the possibility of layoff. At the end of each period of employment, the worker is laid off with known probability $q \in[0,1],{ }^{6}$ in which case he becomes unemployed starting from next period. With probability $1-q$, the worker continues to be employed at wage $w$.

Summing up, the timing of a period $t$ of unemployment is as follows:

1. The worker receives $b_{t}=b_{t}^{U I}+b_{0}$, the utility associated with unemployment, equal to the sum of the monetary value of unemployment benefits and a term representing the psychic value of time when unemployed.

2. The worker decides the amount of search effort $s_{t}$ and pays cost of search $c\left(s_{t}\right)$.

3. With probability $s_{t}$ he then receives a job offer $w$ (drawn from $F$ ).

4. Finally, contingent on receiving an offer, he accepts it or declines it. If he accepts, he is employed with wage $w$ starting from period $t+1$, and faces an exogenous layoff probability $q$ in every period. If no offer is received or the offer is declined, the worker searches again in period $t+1$.

Note that, while the cost function and the wage distribution function are assumed to be invariant over time, we allow the utility associated with unemployment to be a function of the length of the unemployment spell. This is meant to reflect the common feature of the Unemployment Insurance system, where benefits are paid only for a limited amount of time. The model is therefore non-stationary: the optimal amount of search effort and the acceptance/rejection strategy will depend on the current length of the unemployment spell. Finally, we should note

\footnotetext{
${ }^{6}$ The assumption of a fixed separation probability $q$ is somewhat unrealistic, given the well documented declining shape of the hazard function for employment spells. However, an attempt to estimate a model with the separation probability being a declining step function did not yield significantly different results.
} 
that the model is set in partial equilibrium and abstracts from any potential response of firms to the presence of job seekers with hyperbolic preferences.

\subsection{Solution}

For any period $t$, we can write down the maximization problem of the unemployed worker for given continuation payoff $V_{t+1}^{U}$ when unemployed and $V_{t+1}^{E}(w)$ when employed at wage $w$. The worker chooses search effort $s_{t}$ and the wage acceptance policy to solve

$$
\max _{s_{t} \in[0,1]} b_{t}-c\left(s_{t}\right)+\beta \delta\left[s_{t} E_{F}\left\{\max \left(V_{t+1}^{E}(w), V_{t+1}^{U}\right)\right\}+\left(1-s_{t}\right) V_{t+1}^{U}\right],
$$

where the expectation is taken with respect to the distribution of wage offers $F$. Expression (2.3) is easily interpretable: the worker in period $t$ receives benefits $b_{t}$ and pays the cost of search $c\left(s_{t}\right)$. The continuation payoffs are discounted by the factor $\beta \delta$, where $\beta$ is the additional term due to hyperbolic discounting (for the exponential worker, $\beta=1$ ). With probability $s_{t}$ the worker receives a wage offer $w$ that he can then accept - thus obtaining, starting from next period, the continuation payoff from employment $V_{t+1}^{E}(w)$ - or reject, in which case he gains next period the continuation payoff from unemployment, $V_{t+1}^{U}$. With probability $1-s_{t}$, the worker does not find a job and therefore receives $V_{t+1}^{U}$. The continuation payoff from employment at wage $w$, from the perspective of the decision maker in period $t$, is

$$
V^{E}(w)=w+\delta\left[q V_{\text {new }}^{U}+(1-q) V^{E}(w)\right] \cdot{ }^{7}
$$

The worker obtains wage $w$ in period $t+1$; then, with probability $1-q$ he maintains his job and continues to receive wage $w$; with probability $q$ he is laid off, in which case he enters a new spell of unemployment whose value is $V_{\text {new }}^{U}$. The exact specification of $V_{\text {new }}^{U}$ is deferred until later. Note that, from the perspective of self $t$, payoffs from period $t+1$ onwards are discounted exponentially.

To make the model operational, we now assume that, beginning in period $T+1$, the environment is stationary. The only environmental variable that we allow to vary over time is $b_{t}^{U I}$. Once UI benefits are exhausted, the environment becomes stationary: in every period the worker chooses the same intensity of search effort and the same acceptance strategy. In DV-P we give a detailed account of the stationary solution: this includes a proof of existence and uniqueness of the stationary Markov perfect equilibrium in the intrapersonal game played by the hyperbolic individual's different selves.

\footnotetext{
${ }^{7}$ Note that the continuation payoff from employment does not depend on the time one becomes employed. Hence we can omit the time subscript.
} 
The equilibrium solution in the stationary model is characterized by a reservation wage policy. Let $w_{T+1}^{*}$ and $s_{T+1}$ represent, respectively, the reservation wage and the optimal level of search effort in the stationary equilibrium. The reservation wage is:

$$
w_{T+1}^{*}=[1-\delta(1-q)] V_{T+1}^{U}-\delta q V_{n e w}^{U} .
$$

The stationary value of being unemployed, $V_{T+1}^{U}$, and the optimal level of search effort, $s_{T+1}$, can then be found by solving the following system of nonlinear equations:

$$
\begin{aligned}
(1-\delta) V_{T+1}^{U} & =b_{T+1}-c\left(s_{T+1}\right)+\frac{\delta s_{T+1}}{1-\delta(1-q)} Q\left(w_{T+1}^{*}\right) ; \\
c^{\prime}\left(s_{T+1}\right) & =\frac{\beta \delta}{1-\delta(1-q)} Q\left(w_{T+1}^{*}\right),
\end{aligned}
$$

where $Q(x) \equiv \int_{x}^{\infty}(u-x) d F(u)$. Having obtained the solution for the stationary problem, it is straightforward to solve the entire model by backwards induction. The dynamic equations characterizing the solution in periods $t=1, \ldots, T$ are :

$$
\begin{aligned}
w_{t}^{*} & =[1-\delta(1-q)] V_{t+1}^{U}-\delta q V_{\text {new }}^{U} ; \\
V_{t}^{U}-\delta V_{t+1}^{U} & =b_{t}-c\left(s_{t}\right)+\frac{\delta s_{t}}{1-\delta(1-q)} Q\left(w_{t}^{*}\right) ; \\
c^{\prime}\left(s_{t}\right) & =\frac{\beta \delta}{1-\delta(1-q)} Q\left(w_{t}^{*}\right) .
\end{aligned}
$$

To obtain a solution to this model, we need to specify $V_{n e w}^{U}$, the value of becoming unemployed after having been laid off. Given the features of the Unemployment Insurance system, $V_{\text {new }}^{U}$ should depend on the accepted wage and on the duration of the employment spell. However, this would introduce considerable difficulties in calculating the solution. Instead, I make the simplifying assumption that $V_{\text {new }}^{U}=V_{T+1}^{U}$. This implies that a worker is no longer eligible to take UI benefits after his first spell of unemployment. While this assumption is unrealistic for most workers, it is plausible to assume that any bias introduced will not be large. ${ }^{8}$

\footnotetext{
${ }^{8}$ I also experimented with the assumption that $V_{\text {new }}^{U}=V_{1}^{U}$ : in other words, once the worker is laid off he starts a new unemployment spell with all parameters taking on the same values as in the current spell. In this case a state variable is added to the dynamic programming model $\left(V_{1}^{U}\right)$, and the solution involves finding a fixed point to the system

$$
V_{1}^{U}=h\left(V_{1}^{U}\right)
$$

where the function $h$ is defined recursively by equations (2.5)-(2.9) with $V_{\text {new }}^{U}=V_{1}^{U}$. A fixed point did not always exist for all parameter values; where it was possible to obtain a solution, it did not differ substantially from the one obtained assuming $V_{n e w}^{U}=V_{T+1}^{U}$.
} 
Table 1 summarizes the comparative statics for the stationary level of search effort, of reservation wages, and of the implied exit rate from unemployment as functions of the model parameters. Most of the comparative statics results are familiar from the vast literature on job search models (see for example Burdett and Ondrich, 1985). A rise in the utility of unemployment $b$ is associated with lower search effort and higher reservation wages. A location shift in the wage distribution increases the benefits of search, and therefore leads to higher search effort, higher reservation wages, and a higher probability of acceptance. A mean preserving spread in the wage distribution, by raising the probability of drawing a very high wage (whereas a very low wage can always be rejected), raises search effort and reservation wages, while its effect on the probability of acceptance is ambiguous. An increase in the probability of layoff lowers the value of a current job offer, and therefore also lowers the reservation wage and the intensity of search. Its effect on the exit rate from unemployment is ambiguous. Finally a multiplicative increase in the cost function lowers search effort. Since search has become more costly the worker becomes less selective in his job acceptance policy. The global effect of an increase in costs on the exit rate is ambiguous.

In DV-P, we highlighted the relationship between the outcomes of the job search process and the impatience parameters $\beta$ and $\delta$. Impatience has two contrasting effects on the job search process. Impatient individuals dislike all sorts of investment activities, and therefore exert little search effort. On the other hand, once they have a job offer in hand, they prefer to accept it right away rather than wait an additional period for an even better offer. In other words, impatience lowers the probability of receiving, but raises the probability of accepting a job offer. Both impatience parameters in fact operate in the same direction on the intensity of search effort and on the reservation wage. However, the magnitude of the effects differs sharply: the short run discounting parameter $\beta$ operates mainly on the intensity of search effort, while the long-run discounting parameter $\delta$ influences primarily the reservation wage. As a result, higher short-run impatience is associated with longer unemployment spells (the search effect dominates). In DV-P we show that this result requires only a very mild assumption on the wage distribution, which is implied by all log-concave distributions and is comfortably satisfied by other distributions commonly used in the search literature. ${ }^{9}$ By contrast, higher long-run impatience is in general associated with shorter unemployment spells (the reservation wage effect dominates). This result is always true for values of $\delta$ close enough to one. Extensive simulations in DV-P show that for plausible parameterizations of the wage distribution and of the layoff probability the exit rate is decreasing in $\delta$ for all values of the yearly discount factor

\footnotetext{
${ }^{9}$ See DV-P, Proposition 2.
} 
greater than $0.8 .^{10}$ In other words, over the range of values that are considered plausible in the consumption and finance literature, the exit rate is decreasing in $\delta$.

For intuition on this result, consider the two separate decisions making up the search process. The decision on intensity of search effort involves a trade-off between the present costs of searching and benefits that will start to materialize in the near future, once an offer is accepted. This time span is relatively short: in the United States most spells end in less than 30 weeks. Over this limited time horizon, short-run impatience matters the most. On the other hand, the reservation wage decision involves a comparison of long-term consequences, once an offer is received: the worker chooses whether to accept the wage or wait for an even better offer. Since most employment spells last for more than a year, the worker is making a choice for the long run. Therefore, the reservation wage is mainly affected by the degree of long-run discounting. The distinct role played by $\beta$ and $\delta$ in the job search process is important for identification issues. Despite the fact that both $\beta$ and $\delta$ represent the degree of preferences for the present versus the future, it is possible to identify them separately. The above discussion suggests that, to a first approximation, data on reservation wages alone may be sufficient to identify $\delta$, and data on search intensity may be sufficient to identify $\beta$.

\section{Estimation}

I now turn to the structural estimation of the job search model, using data on the duration of unemployment spells and on re-employment wages, as in the classic works of Wolpin (1987), and van den Berg (1990). My work is new in that it allows for hyperbolic preferences, and centers its attention on the discounting parameters. I also attempt to estimate the parameters of the cost of search function, a feature absent from models with an exogenous offer arrival rate. As in van den Berg, I introduce non-stationarity by allowing for UI benefits to run out after a limited amount of time. I estimate the model separately for three groups of workers, classified by their wage prior to the unemployment spell. In this way, I am able to control for observed heterogeneity along one very important dimension. In addition, in the basic specification I will allow variation in parameters by AFQT scores and marital status within each wage group. ${ }^{11}$ Finally, I incorporate unobserved heterogeneity in the form of a finite mixture distribution for the model parameters. This will help to capture the negative duration dependence in unemployment spells typically observed in this type of data. In section $4.4 \mathrm{I}$

\footnotetext{
${ }^{10}$ See DV-P, Table 1.

${ }^{11} \mathrm{AFQT}$ and marital status were chosen as the explanatory variables since it appeared that the most significant variation in exit rates and re-employment wages occurred along these lines.
} 
consider alternative forms in which observed heterogeneity may affect the parameters of the model.

\subsection{The likelihood function}

The data consists of observations on the length of unemployment spells in weeks, $T_{i}$, on reemployment wages $w_{i}$, and on a set of individual characteristics $X_{i}$ for a sample of individuals $i=1,2, \ldots, N$. Assume for the moment that there are no censored spells and that the reemployment wage is observed for every individual in the sample. I take the week as the time unit, and I denote by $h_{t}\left(\theta, X_{i}\right) \equiv s_{t}\left(\theta, X_{i}\right)\left[1-F_{\theta}\left(w_{t}^{*}\left(\theta, X_{i}\right)\right)\right]$ the exit rate from unemployment in week $t$ as a function of the model parameters $\theta$ and of individual characteristics $X_{i}$. The exit rate in week $t$ is simply the product of the probability of receiving an offer in week $t$, $s_{t}\left(\theta, X_{i}\right)$, and the probability that this offer exceeds the reservation wage in week $t, w_{t}^{*}\left(\theta, X_{i}\right)$. The values of $s_{t}\left(\theta, X_{i}\right)$ and $w_{t}^{*}\left(\theta, X_{i}\right)$ are obtained from the solution of the dynamic programming problem (2.3). The likelihood contribution of individual $i$ can be written (omitting the dependency on $X_{i}$ for notational convenience) as

$$
\begin{aligned}
\mathcal{L}_{i}(\theta)= & \left(\prod_{\tau=1}^{T_{i}-1}\left[1-h_{\tau}(\theta)\right]\right) \times\left[h_{T_{i}}(\theta)\right] \times\left(\frac{f_{\theta}\left(w_{i}\right)}{1-F_{\theta}\left[w_{T_{i}}^{*}(\theta)\right]} 1\left(w_{i} \geq w_{T_{i}}^{*}\right)\right) \\
= & \left(\prod_{\tau=1}^{T_{i}-1}\left\{1-s_{\tau}(\theta)\left[1-F_{\theta}\left(w_{\tau}^{*}(\theta)\right)\right]\right\}\right) \times \\
& \times\left\{s_{T_{i}}(\theta)\left[1-F\left(w_{T_{i}}^{*}(\theta)\right)\right]\right\} \times \\
& \times\left(\frac{f_{\theta}\left(w_{i}\right)}{1-F_{\theta}\left[w_{T_{i}}^{*}(\theta)\right]} 1\left(w_{i} \geq w_{T_{i}}^{*}\right)\right) .
\end{aligned}
$$

The first term represents the probability that individual $i$ does not exit unemployment in any of the periods from $t=1$ to $T_{i}-1$; the second term represents the probability that the individual finds an acceptable offer in period $T_{i}$; the last term is the probability density of observing a re-employment wage $w_{i}$, conditional on that wage being greater than the reservation wage $w_{T_{i}}^{*}$. Note that the likelihood is well-defined only if the actual wage is greater than the reservation wage for the week in which the worker actually finds work. This can result in extreme sensitivity of the estimates to a few outliers. To prevent this possibility, I make the plausible assumption, as in Wolpin (1987), that wages are in fact measured with error: let $\tilde{w}_{i}$ be the true wage, and 
$w_{i}^{o b s}$ be the observed wage. Then the third element becomes

$$
f\left(w_{i}^{o b s} \mid \tilde{w}_{i}>w_{T_{i}}^{*}(\theta)\right)=\frac{P\left(\tilde{w}_{i} \geq w_{T_{i}}^{*}(\theta) \mid w_{i}^{o b s}\right) f\left(w_{i}^{o b s}\right)}{P\left(\tilde{w}_{i} \geq w_{T_{i}}^{*}(\theta)\right)} .
$$

With a convenient specification of the joint distribution of $w_{i}^{o b s}$ and $\tilde{w}_{i}$, this term can be easily calculated.

Censored observations and missing wage data are easily incorporated in this setting. Let $C_{i}$ be a dummy variable indicating whether the observed unemployment spell is complete, and let $E_{i}$ be a dummy indicating whether the re-employment wage is observed. ${ }^{12}$ The likelihood then becomes:

$$
\begin{aligned}
\mathcal{L}_{i}(\theta)= & \left\{\left(\prod_{\tau=1}^{T_{i}-1}\left[1-h_{\tau}(\theta)\right]\right)\left[h_{T_{i}}(\theta)\right]\left(\frac{P\left(\tilde{w}_{i} \geq w_{T_{i}}^{*}(\theta) \mid w_{i}^{o b s}\right) f\left(w_{i}^{o b s}\right)}{P\left(\tilde{w}_{i} \geq w_{T_{i}}^{*}(\theta)\right)}\right)\right\}^{C_{i} E_{i}} \times \\
& \times\left\{\left(\prod_{\tau=1}^{T_{i}-1}\left[1-h_{\tau}(\theta)\right]\right)\left[h_{T_{i}}(\theta)\right]\right\}^{C_{i}\left(1-E_{i}\right)} \times\left\{\prod_{\tau=1}^{T_{i}}\left[1-h_{\tau}(\theta)\right]\right\}^{1-C_{i}} \times
\end{aligned}
$$

Summing over individuals, and taking logs, yields the following log-likelihood function:

$$
\begin{aligned}
L(\theta)= & \sum_{i=1}^{N} \sum_{\tau=1}^{T_{i}-1} \log \left[1-h_{\tau}(\theta)\right]+ \\
& \sum_{i: C_{i}=1} \log \left[h_{T_{i}}(\theta)\right]+ \\
& \sum_{i: C_{i}=0} \log \left[1-h_{T_{i}}(\theta)\right]+ \\
& \sum_{i: C_{i}=1, E_{i}=1} \log \frac{P\left(\tilde{w}_{i} \geq w_{T_{i}}^{*}(\theta) \mid w_{i}^{o b s}\right) f\left(w_{i}^{o b s}\right)}{P\left(\tilde{w}_{i} \geq w_{T_{i}}^{*}(\theta)\right)} .
\end{aligned}
$$

The dependency on observed individual characteristics is made explicit by letting $h_{t}\left(\theta, X_{i}\right)=$ $\tilde{h}_{t}\left(\theta_{1} ; \theta_{2}^{\prime} X_{i}\right)$ : in practice, to keep the dimension of the problem manageable, the control variables will be a set of indicators, so that the hazard rate can be effectively be written as $\tilde{h}_{t}\left(\theta_{1}, \theta_{2 i}\right)$. Finally, one can add unobserved heterogeneity to the model by specifying a mix-

\footnotetext{
${ }^{12}$ In my sample there are observations with missing re-employment wage data, even if the unemployment spell is complete.
} 
ture distribution with discrete finite support for the parameter vector $\theta$ :

$$
\theta=\left\{\begin{array}{ccc}
\xi_{1} & p_{1} & \\
\xi_{2} & p_{2} \\
& \vdots & \\
\xi_{K} & p_{K}
\end{array} \quad \sum_{k=1}^{K} p_{k}=1\right.
$$

The log-likelihood function for the model with unobserved heterogeneity becomes

$$
\begin{aligned}
L_{p}(\theta) & =\log \int \exp [L(\theta)] d p(\theta) \\
& =\log \sum_{k=1}^{K} p_{k} \exp \left[L\left(\xi_{k}\right)\right] .
\end{aligned}
$$

\subsection{Functional form specification}

In order to solve the model and to identify its parameters, one needs to specify functional forms for the cost function and the wage distribution function, and set values for those parameters that are not directly estimated. The choice of functional forms is dictated by empirical plausibility and by computational convenience.

The value of time when unemployed. I model the value of time when unemployed as the sum of the monetary value of UI benefits and a time invariant component $b_{0}$.

$$
\begin{aligned}
b_{t} & =b_{t}^{U I}+b_{0} \\
b_{t}^{U I} & = \begin{cases}b^{U I} & \text { if } t \leq T \\
0 & \text { if } t>T\end{cases}
\end{aligned}
$$

The second component can be thought of as either positive (utility of leisure) or negative (stigma associated with unemployment). It enters as an unknown parameter in the likelihood function. Consistent with the UI system in most states, I assume that benefits are received for $T=26$ weeks. The monetary value of unemployment benefits $b^{U I}$ is fixed at the average value of actual benefits observed in the population of interest.

The wage distribution. I assume that actual wages $\tilde{w}_{i}$ are drawn from a log-normal distribution with parameters $\mu_{i}$ and $\sigma_{i}$. The moments of the log wage distribution are assumed to depend on observed characteristics $X_{i}$. The vector $X_{i}$ consists of six indicator variables indicating the possible combinations of marital status and three AFQT dummies (high, medium, 
and low). Observed wages $w_{i}^{o b s}$ are equal to actual wages times a multiplicative error term with a log-normal distribution, so that

$$
\log w_{i}^{o b s}=\log \tilde{w}_{i}+u_{i}
$$

and

$$
\left(\begin{array}{c}
\log \tilde{w}_{i} \\
u_{i}
\end{array}\right) \sim N\left[\left(\begin{array}{c}
\mu_{i} \\
0
\end{array}\right),\left(\begin{array}{cc}
\sigma_{i}^{2} & 0 \\
0 & \sigma_{u}^{2}
\end{array}\right)\right] .
$$

This specification implies that log wages are measured with a white noise measurement error: this assumption is plausible if one considers that many of the other characteristics of a job, pecuniary and not, are not observed in our data. The joint normality of actual log wages and the error term implies joint normality between actual log wages and observed log wages. Then, using the properties of the multivariate normal distribution, we can derive

$$
\begin{aligned}
f\left(w_{i}^{o b s} \mid \tilde{w}_{i}>w_{T_{i}}^{*}(\theta)\right) & =\frac{P\left(\tilde{w}_{i} \geq w_{T_{i}}^{*}(\theta) \mid w_{i}^{o b s}\right) f\left(w_{i}^{o b s}\right)}{P\left(\tilde{w}_{i} \geq w_{T_{i}}^{*}(\theta)\right)} \\
& =\frac{\left[1-\Phi\left(\frac{\log w_{T_{i}}^{*}-a_{1 i}-a_{2 i} \log w_{i}^{o b s}}{v_{i}}\right)\right]\left(\frac{1}{w_{i}^{o b s} \sqrt{\sigma_{i}^{2}+\sigma_{u}^{2}}} \phi\left(\frac{\log \left(w_{i}^{o b s}\right)-\mu_{i}}{\sqrt{\sigma_{i}^{2}+\sigma_{u}^{2}}}\right)\right)}{1-\Phi\left(\frac{\log w_{T_{i}}^{*}-\mu_{i}}{\sigma_{i}}\right)},
\end{aligned}
$$

with $a_{2 i}=\frac{\sigma_{i}^{2}}{\sigma_{i}^{2}+\sigma_{u}^{2}}, a_{1 i}=\mu_{i}\left(1-a_{2 i}\right)$, and $v_{i}^{2}=\sigma_{i}^{2}-a_{2 i}^{2}\left(\sigma_{i}^{2}+\sigma_{u}^{2}\right)$, and $\Phi(\cdot)$ and $\phi(\cdot)$ represent the standard normal cumulative distribution function and probability density function respectively. The parameters $\mu_{i}, \sigma_{i}$, and $\sigma_{u}$ all need to be estimated from the data.

The cost function. A convenient functional form that captures the main features of the cost function is

$$
c_{i}(s)=k_{i} s^{1+\eta}, \quad \eta>0 .
$$

The parameter $\eta$ represents the degree of convexity of the cost function. It is also equal to the (constant) elasticity of marginal cost of search with respect to effort: the higher the value of $\eta$, the higher the proportional increase in the marginal cost of search for a given percentage increase in search effort. The parameter $k_{i}$ represents the scale of the cost function: it tells us how costly it would be to obtain a job with probability one. I allow this parameter to depend on observed characteristics $X_{i}$.

The parameter $\eta$ is estimated using data from the 1981 wave of the NLSY that provides detailed information on the search activities of employed and unemployed youth. ${ }^{13}$ I consider

\footnotetext{
${ }^{13}$ This is the same data used in Holzer (1988).
} 
an extension of the model described in the text in which workers have already chosen the optimal probability of receiving an offer $s$, and must now decide how to optimally allocate their time between alternative search methods. Assume that there are $K$ different methods that can be used to generate offers. Once the worker has chosen $s$, he must decide how intensively to use each method of search. Assume also that the effort exerted in this period has no bearing on the probability of receiving an offer in any of the latter periods, and that each search method generates offers from the same wage distribution. These assumptions make the model effectively static, and allow us to abstract from the issues of time discounting.

The worker's problem becomes one of optimally choosing intensity of search for each method so as to minimize search costs, subject to the constraint that the probability of receiving an offer be at least $s$. Formally, the problem is

$$
\begin{array}{ll} 
& \min _{X_{1}, \ldots, X_{K} ;} \sum_{j=1}^{K} c_{j} X_{j} \\
\text { s.t. } \quad: \quad & P\left(X_{1}, \ldots, X_{K}\right) \geq s \\
X_{j} \quad \geq & 0, \quad j=1, \ldots, K,
\end{array}
$$

where $X_{j}$ is the amount of hours devoted to search using method $j$, and $c_{j}$ is the cost of one hour of search using method $j$. In the Appendix, I show that an appropriate choice of functional form for $P\left(X_{1}, X_{2}, \ldots, X_{K}\right)$ yields a closed form solution for the optimal hours of search for each method, and these in turn generate a cost of search function of the form

$$
\tilde{c}(s)=A(1-s)^{-\varepsilon}-B,
$$

where $A, B$, and $\varepsilon$ are functions of productivity and the cost of each search method. The productivity of search parameters are estimated by maximum likelihood, using data on whether each search method resulted in a job offer. Then one can use the first order conditions to back out the cost of search parameters $c_{j}$, up to a constant of proportionality (details in Appendix 1). Given $\alpha$ and $c$, one can evaluate the cost function $\tilde{c}(s)$ at various levels of search intensity $s$. These estimated values are then used to estimate the parameters of the constant marginal cost elasticity cost function, $c(s)=k s^{1+\eta}$, used in the dynamic programming model. The estimation is performed by running a weighted least squares regression of $\log \tilde{c}(s)$ on $\log s$, where the weights are proportional to a normal density centered at the estimated mean probability of receiving a job offer. ${ }^{14}$ The results of this regression are

\footnotetext{
${ }^{14}$ The regression is weighted because the actual cost function $\tilde{c}(s)$ and the approximate cost function $c(s)$ can differ substantially at extreme values of $s$. Therefore we give more weight to those values of $s$ that have higher density empirically.
} 


$$
\log \tilde{c}(s)=4.412+1.408 \log s
$$

The estimated constant marginal cost elasticity $\eta$ is then equal to the coefficient on $\log s$ minus one. This gives rise to the benchmark value of $\eta=0.4$.

One can then use this value and information on weekly offer arrival probabilities to gauge the magnitude of $k$. For example, if one believes that the weekly cost of search is roughly of the same order of magnitude as the weekly wage, say $\$ 250,{ }^{15}$ then a weekly offer probability of 0.1 and the estimated value of $\eta$ imply a value for $k$ of $250 /(0.1)^{1.4} \approx 6,300$. If the weekly offer probability is instead 0.05 , the implied value for $k$ is approximately 16,600 .

Unobserved Heterogeneity. I introduce unobserved heterogeneity by assuming that the population is composed of two unobserved types, in proportions $p$ and $1-p$. Type 1 workers (in proportion $p$ ) have cost of search parameter $k_{i}+\Delta k$ and face a wage offer distribution with mean $\mu_{i}+\Delta \mu$. Type 2 workers have cost of search parameter $k_{i}$ and face a wage offer distribution with mean $\mu_{i}$. The parameter $\Delta k$ is restricted to be positive, indicating that type 1 workers can be viewed as low cost of search workers, while type 2 workers are high cost workers. No restrictions are placed on $\Delta \mu{ }^{16}$

To illustrate behavior of the key variables in the solution, I depict in Figure 1 the dynamic evolution of the probability of exiting unemployment $h(t)$ and of the expected re-employment wage $E\left(w \mid w>w_{t}^{*}\right)$. The figure shows the dynamics of these variables for low cost workers, high cost workers, and for the aggregate of the two types. For both types the exit rate is monotonically increasing, and the expected re-employment wage is monotonically decreasing. When we look at the aggregate variables, we see that the introduction of heterogeneity generates a non-monotonic shape for the hazard function, with a moderate spike at the time of exhaustion of UI benefits.

\footnotetext{
${ }^{15}$ In 1983 dollars.

${ }^{16} \mathrm{It}$ is not necessary to interpret the mixture distribution as actual heterogeneity. One may also view the sample as composed of homogeneous workers, who own a fixed stock of two types of "search capital" at the beginning of their unemployment spell: one type of capital generates job offers at low cost but depreciates rapidly (think of this as search through friends and relatives); the second type of capital generates job offers at high cost but depreciates more slowly (search through formal channels).
} 


\subsection{Identification}

Using the functional form specification described above, there are 27 unknown parameters that need to be estimated; the two discounting parameters, $\beta$ and $\delta ;^{17}$ the value of time when unemployed, $b_{0}$; the parameter vectors of the wage distribution $\boldsymbol{\mu}$ and $\boldsymbol{\sigma}$ (six elements each); the standard deviation of the measurement error in log wages, $\sigma_{u}$; the cost of search parameter vector $\mathbf{k}$ (six elements); and the parameters of the heterogeneity distribution, $\Delta k, \Delta \mu$ and $p$. In addition, one must specify a value for the marginal cost elasticity $\eta$ and for the probability of layoff $q$. The marginal cost elasticity $\eta$ is set at 0.4 , following the calculations described in Section 3.2. I estimate $q$ separately for the three wage groups using employment spells from the NLSY from 1985 to 1996. This results in $q=0.0111, q=0.0105$, and $q=0.0087$ for the low, medium, and high wage groups respectively.

I give here a heuristic description of the identification strategy. Consider first an unrestricted model in which workers choose search effort and reservation wages, possibly not based on any optimality principle. The parameters of this model, the reservation wage and the probability of receiving an offer in any period, are identified using data on reservation wages and duration data alone. In any period $t$, the reservation wage $w_{t}^{*}$ can be estimated by the minimum observed wage for those who exit unemployment in that period. Given the assumption of stationarity from period $T$ onwards, one can estimate the stationary reservation wage as the minimum of all observed wages among those with unemployment spells longer than $T$ weeks. The distribution of observed wages for those unemployed longer than $T$ periods, together with the reservation wage, identifies the parameters of the wage distribution. Having identified the parameters of the wage distribution and the reservation wages, it is easy to calculate the conditional probability of acceptance in each period. ${ }^{18}$ This, together with the data on the duration of unemployment spells, helps to identify the level of search effort in every period, $s_{t}$ : in the model, $s_{t}$ is simply the probability of receiving a job offer, equal to the ratio between the exit rate from unemployment and the probability of acceptance. Having identified $s_{t}$ and $w_{t}^{*}$, identification of the other model parameters follows in principle by imposing the restrictions implied by the dynamic optimization problem.

In practice, however, it may be difficult to separately identify all the model parameters. I should reiterate, though, that the discounting parameters $\beta$ and $\delta$ can be separately identified

\footnotetext{
${ }^{17}$ The time unit for the model is a week, but, for convenience of interpretation, I choose to present all the results in terms of the yearly discount factor, which, with slight abuse of notation, is denoted by $\delta$.

${ }^{18}$ The conditional probability of acceptance is the probability of accepting a wage offer, conditional on having received one.
} 
when the other model parameters are known. As discussed in Section 2.3, the two parameters play fundamentally distinct roles in the job search process: the short-run discount factor $\beta$ affects mainly the search effort decision, a decision involving a trade-off between immediate costs and benefits that materialize in the near future; on the other hand, the reservation wage decision involves primarily a trade-off between payoffs to be received at two different points in the more distant future, and therefore is nearly unaffected by $\beta$ (it is affected by $\beta$, but only indirectly through the effect of $\beta$ on the continuation payoff). This suggests that, to a first approximation, one can use data on reservation wages to identify $\delta$. Then, for a known value of $\delta$, it is straightforward to identify $\beta$ using duration data.

To better illustrate this point, I generate some simulated unemployment histories, ${ }^{19}$ and then study the behavior of the likelihood function assuming that data is available either on both duration and re-employment wages, or on duration alone. Figure 2a shows the log-likelihood and likelihood profiles as functions of $\beta$ assuming either complete or incomplete data. ${ }^{20}$ It is apparent that data on wages does not contribute significantly to the identification of $\beta$. On the other hand, the identification problem stands out when we analyze the likelihood profiles as functions of the long-term discount factor $\delta$. When data on wages is not available, the loglikelihood as a function of $\delta$ is essentially flat, and the likelihood is substantially spread out; incorporating information on accepted wages allows us to identify $\delta$.

\section{Results}

\subsection{Data}

My data contains information on the duration of unemployment spells and re-employment wages for males in the NLSY. I use the Work History files to construct a week by week account of every male worker's labor force status from 1978 to 1996. A worker is defined to be unemployed if he is out of a job but willing to work. I classify as unemployment spells all periods of nonemployment in which at least some active search took place. This measure differs from the conventional definition in that a worker who does not actively search during the entire spell can still be classified as unemployed. The re-employment wage is taken to be the average weekly wage (in 1983 dollars) in the first job after the end of the unemployment

\footnotetext{
${ }^{19}$ I generated 5000 simulated unemployment histories according to the model described in Section 2. The true values of $\beta$ and $\delta$ were 0.4833 and 0.95 respectively. All the other parameter values were set at their maximum likelihood estimates for the medium wage sample (Table 3 ).

${ }^{20}$ The likelihood and log-likelihood are normalized to facilitate comparison between the complete and incomplete data functions.
} 
spell.

I retain only those spells that were reported in 1985 or later by male respondents with no health problems, who were not part of the military subsample, and were not enrolled in school. This ensures that my sample of spells includes mainly workers with strong attachment to the labor force. In addition, since I am interested in estimating a model with time-varying unemployment benefits, I retain only spells in which Unemployment Insurance was received for at least one week. In addition, I discard observations where the logarithm of the re-employment wage is more than three and a half standard deviations below or above the mean, or with missing data on the wage prior to the unemployment spell. This leaves me with 1797 observations, representing 1008 different individuals. For the purposes of this paper, I assume that different spells by the same individual can be treated as independent spells.

The empirical analysis is performed separately for three groups classified by their previous earnings. The low earnings group includes workers in the bottom quartile of the sample distribution of previous weekly wages; the medium earnings group includes individuals in the middle two quartiles; the top earnings group includes individuals in the top quartile. Summary statistics on the duration of unemployment spells, on re-employment wages, and on a set of demographic characteristics are presented in Table 2.

There are marked differences between the three groups in all the variables. Workers with higher previous earnings have shorter unemployment spells and are employed at higher wages at the end of their spell. Moreover, as we move across the earnings distribution, we find workers who are older, more educated, more likely to be married, and with higher levels of cognitive ability as measured by the AFQT score. By contrast, the replacement rate falls from about $70 \%$ of the previous wage for low wage workers to about $30 \%$ for high wage workers. Overall there is substantial heterogeneity in the sample.

\subsection{Implementation}

The theoretical model imposes restrictions on the range of possible parameter values. For example the long term discount factor $\delta$ must be between 0 than 1 . Hyperbolic discounting posits that the short-run discount factor $\beta$ be smaller than unity, but the model does allow values of $\beta$ greater than one, provided that $\beta$ is smaller than $1+\eta \cdot{ }^{21}$ To ensure that

\footnotetext{
${ }^{21}$ Solution of the stationary system in (2.5) and (2.6) yields the following two equations in two unknowns, $w^{*}$ and $s^{*}$ :$$
\frac{Q\left(w^{*}\right)}{\left(w^{*}-b\right)^{1 /(1+\eta)}}=\left(\frac{k}{\beta}\right)^{1 /(1+\eta)} \frac{(1+\eta)(1-\delta(1-q))}{\delta(1+\eta-\beta)^{1 /(1+\eta)}}
$$ 
the optimization algorithm does not wander off testing nonsensical values, I apply a logistic transformation to all the model parameters: for any structural parameter $\theta_{j}$ restricted to the interval $\left(c_{1}, c_{2}\right)$, I define $\gamma_{j}=\log \left[\left(\theta_{j}-c_{1}\right) /\left(c_{2}-\theta_{j}\right)\right]$. I then reparameterize the likelihood in terms of the $\gamma$ 's, which are allowed to take on any values on the real line. The maximum likelihood estimate for the original structural parameter $\theta_{j}$ is easily obtained as $\hat{\theta}_{j}^{M L}=\left[c_{1}+c_{2} \exp \left(\hat{\gamma}_{j}^{M L}\right)\right] /\left[1+\exp \left(\hat{\gamma}_{j}^{M L}\right)\right]$, and standard errors for $\theta_{j}$ are calculated using the delta method. Standard errors for $\gamma$ are obtained using the outer product gradient method.

The model is estimated using a pre-conditioned conjugate gradient algorithm with numerical derivatives from the NAG Fortran library. Convergence to the maximum and calculation of standard errors took approximately 15 minutes on a dual processor 800 MhZ Pentium computer. To ensure that the algorithm had in fact converged to a global maximum, the optimization procedure was repeated with different starting values. The results of the structural estimation are given below.

\subsection{Structural parameter estimates}

In Table 3 I present parameter estimates and standard errors for the structural parameters in the three subsamples.

The first observation to be made is that the estimate of the hyperbolic discounting parameter $\beta$ increases as we move from the low to the high end of the wage distribution. For low and medium wage workers we find a high degree of present bias, whereas for high wage workers the degree of short run impatience is relatively small. The point estimates for $\beta$ in the low and medium wage samples are somewhat lower than those found in the experimental literature, (Thaler, 1981; Benzion, Rapoport and Yagil, 1989; Kirby, 1997), but not totally implausible, especially if we take into account that the experimental subjects are likely not representative of the whole population. Fang and Silverman (2002) estimate a similar model of job search for women receiving welfare, and they estimate $\beta$ equal to 0.61 , with quite a large standard error. The ranking of $\beta$ across the distribution of previous wages is not surprising: we expect workers who are able to delay gratification and have relatively little self-control problems to be rewarded in the labor market. Using a Wald test, the null hypothesis of exponential discounting is rejected in the low and medium wage samples, and not rejected in the high wage sample.

$$
s^{*}=\left[\frac{\beta\left(w^{*}-b\right)}{k(1+\eta-\beta)}\right]^{\frac{1}{1+\eta}} .
$$

The solution is well defined for $\beta<1+\eta$. 
It is also possible to test the restriction $\beta=1$ using a likelihood ratio test. The results of these tests are reported at the bottom of Table 3. In the low and medium wage samples, the exponential model is soundly rejected, whereas the evidence in the high wage sample is ambiguous with a p-value of 0.08 .

The estimate of the degree of long-run discounting $\delta$ lies extremely close to the boundary of the parameter space, and its confidence interval is essentially uninformative. Interestingly, we find that the value of time when unemployed $b_{0}$ is substantially negative in all three samples. This implies that the mere fact of being unemployed bears disutility. This is consistent with the literature on subjective measures of happiness (Oswald, 1997; Winkelmann and Winkelmann, 1998): these studies find that unemployment has a universally negative effect on subjective measures of well-being, which goes well beyond that predicted by the income drop associated with joblessness. Moreover, other structural estimates of the job search model also find a similar result: van den Berg (1990) reports that workers "dislike being unemployed ... for nonpecuniary reasons," 22 and Wolpin(1987) estimates a positive weekly cost of search ranging from 104 to 223 dollars. Note also that the disutility is greater as we move up the wage distribution: the psychic cost of being unemployed is larger for workers who experience unemployment (either personally or through acquaintances) relatively rarely.

The high estimated value of $\delta$ has implications for the other model parameters: in particular, agents, being extremely patient in the long run, will reject a large proportion of offered wages. This is reflected in the estimates of $\boldsymbol{\mu}$ : the estimated mean of the log wage distribution lies between 50 and $100 \log$ points below the mean of observed log wages in all population subgroups, indicating that a sizeable fraction of wages are rejected. Similarly, the estimated standard deviation of the log wage distribution is larger than the observed standard deviation in all subsamples. Measurement error accounts for only a small fraction of the total variation in log wages. Altogether, there is substantial heterogeneity in the parameters of the wage distribution across population subgroups, for all three samples.

The cost of search parameter vector $\mathbf{k}$ follows a general pattern in all three subsamples, with costs of search being the highest for unmarried workers with low AFQT and the lowest for married workers with high AFQT. It is conceivable that low-skill workers do indeed face a higher cost of obtaining a job offer with a given probability. Similarly, the result on marital status can be explained by the fact that married workers have a broader network of acquaintances that may facilitate the job search process. It is worth noting that the pattern of the parameters is the same pattern that appears in the distribution of unemployment duration (which is correlated

\footnotetext{
${ }^{22}$ van den Berg (1990), page 270.
} 
negatively with cognitive ability and marriage): it appears that the cost of search parameter is governing most of the variation in exit rates across groups.

\subsection{Alternative forms of heterogeneity}

A natural question that arises is whether the results are robust to different specifications of the structural model. In particular, the basic specification adopts a very simple structure for the way in which observed and unobserved heterogeneity affect the model parameters. I now consider three alternative forms of heterogeneity. In Alternative 1, I let the distribution of the unobserved component have three mass points rather than two. In Alternative 2, I add race as one of the explanatory variables for the parameters of the cost of search function and of the wage offer distribution, while maintaining a two-point distribution for unobserved heterogeneity. The model estimated in Table 3 is nested in these first two alternative models, so that we can perform likelihood ratio tests to assess whether the alternatives significantly improve the fit of the model.

In Alternative 3 I follow a substantially different approach. I allow observed covariates to be correlated with type probabilities: I eliminate the direct dependence of $\mu$ and $k$ on $X_{i}$, and instead specify that the probability that individual $i$ is of type $j$ is equal to

$$
P(\text { individual } i \text { is of type } j)=\frac{e^{\alpha_{j}^{\prime} X_{i}}}{\sum_{k} e^{\alpha_{k}^{\prime} X_{i}}} .
$$

In practice, I let there be only two types, and $X_{i}$ is a twelve-element vector representing all the possible combinations of AFQT scores (low, medium and high), marital status and race. These alternative models were estimated for the medium wage sample only, and the results are presented in Table 4.

Alternatives 1 and 2 significantly improve the fit of the model compared to Table 3 , as can be seen from the likelihood ratio test statistics and its associated p-values. However, in terms of the point estimate of $\beta$, there is little difference between the two models and the basic specification. While the standard errors around $\beta$ are lower in the richer specifications, the likelihood ratio test statistic for the null hypothesis of exponential discounting gives somewhat weaker results, and in fact we cannot always reject the null hypothesis of exponential discounting. The third alternative, which adopts a substantially different framework for incorporating observed and unobserved heterogeneity, yields a slightly larger point estimate for the short-run discounting parameter, with a relatively large standard error. To summarize, introducing richer structures for the effect of observables and unobservables on the model parameters does not substantially affect our estimate of the degree of present bias, but comes at a cost of a loss in precision. 


\subsection{Predicted values}

It is worthwhile to analyze how the various specifications compare in terms of predicted values for the variables of interest. I use the basic specification of the model, discussed in Table 3. The results are presented in Table 5. I compare the mean values for duration and reemployment wages in the sample to those predicted by the model in the three subsamples. ${ }^{23}$ Likewise, I present predicted values for the expected weekly acceptance probability, and the expected weekly cost of search in the final week of unemployment. All the models do a fairly good job of matching the average re-employment wage. The expected unemployment duration is consistently overestimated, but this was to be expected, given that in the calculation of predicted unemployment duration we give positive weight to extremely long spells that would be censored in the actual data.

The predicted values for the unobserved quantities raise some questions. As noted earlier, the very high degree of long run patience implies that workers are quite selective in their job acceptance strategy. Previous research has estimated the acceptance probability at around 60 percent (Blau and Robins, 1990), substantially higher than my estimate of 14 to 26 percent. Moreover, the estimated cost of search in the high wage sample, equal to more than one and a half times the re-employment wage, seems too high. These findings suggest that it may be helpful to use outside sources of information to place prior restrictions on functions of the structural parameters. The use of an informative prior for a well-defined quantity such as the acceptance probability is easily justifiable. This strategy is appealing in that it may become possible to learn more about parameters that are poorly identified in the likelihood.

\subsection{Dynamics}

The expected values in Table 5 hide a rich set of dynamic behavior in the model. Table 6 and Figures 3a-3c show the dynamic evolution of several variables of interest over the course of an unemployment spell. Dynamics in the model (see Figure 1) are generated by the limited availability of unemployment insurance, and by the changing composition of the sample as the unemployment spell progresses: workers with an unobserved low cost of search exit unemployment much more frequently, so that the pool of workers who are still unemployed is increasingly dominated by the high cost individuals. These two forces operate in opposite

\footnotetext{
${ }^{23}$ The predicted values are the expectations of the relevant variables, evaluated analytically at the maximum likelihood estimates, and integrated over the distribution of both observed and unobserved types. See Appendix B for details.
} 
directions on the exit rate from unemployment. As benefits run out, workers increase the intensity of search and begin to accept a larger fraction of wage offers, so that, for a given worker type, the exit rate increases in the first 26 weeks. On the other hand, the aggregate exit rate decreases as the sample is increasingly dominated by high cost types with low exit rates. The expected re-employment wage, by contrast, decreases monotonically: as the time of benefit exhaustion approaches both types of workers set a lower reservation wage, and, because high cost workers have lower reservation wages, the aggregate expected re-employment wage also declines as more and more low-cost workers exit unemployment.

Figures 3a-3c show that in all three samples the model does a good job at fitting the survivor function, even though it cannot capture the high week to week variation in exit rates and expected re-employment wages. In the low and medium wage samples the exit rate is initially flat and begins to rise as week 26 (the time of exhaustion of UI benefits) approaches. In the high wage sample the exit rate falls monotonically.

The results of Table 6 show that most of the difference in exit rates between high and low wage workers can be attributed to differences in (endogenously determined) offer arrival rates. In fact, conditional on receiving offers, low-wage workers accept a higher proportion of wages, but have longer unemployment spells because of low offer probabilities. This finding is in accordance with much of the previous literature, which finds that variation in offer arrival rates plays a much larger role in explaining unemployment duration than variation in reservation wages (see Devine and Kiefer, 1991). On the other hand, in the comparison between medium wage and low wage workers, we see that the former have higher exit rates because of higher acceptance probabilities.

We see that predicted acceptance probabilities are quite low during the first few weeks of the spell, ranging from 12 to 20 percent. In the low and medium wage samples, the proportion of acceptable offers rises to 34-50 percent after one year, and to 77 to 90 percent after two years. This simply reflects the fact that after two years the sample is composed almost entirely of high-cost types who accept nearly any offer. In the high wage sample, the acceptance probability remains at very low levels even after two years.

The cost of search behaves in a very non-linear fashion: in the low and medium wage samples it increases in the first 26 weeks, and then decreases; in the high wage sample it dips in the first few weeks, then rises up to week 26 , and then decreases. This pattern is explained by the fact that as the time of benefit exhaustion approaches, all worker types search more intensively, driving up search costs; but this effect is offset by the increasing proportion of high cost types, who search less intensively and actually end up paying a lower overall cost of search. 


\section{Policy Evaluation}

One of the main advantages of structural estimation is that it allows one to simulate the effects of different policy interventions in a behaviorally consistent manner. In addition, it is possible evaluate the effects of a given policy on outcomes that are not always observable in other contexts: for example, by fully specifying the agent's preferences and assuming that the agent follows an optimal strategy, we can carry out welfare comparisons between alternative policies. In our setting, this can be particularly important, because a hyperbolic agent's dynamic inconsistency may imply that some policy intervention can raise his or her long-term welfare. Alternatively, one may wish to assess whether the estimated effect of a given policy is sensitive to the assumption made about intertemporal preferences.

For a range of different policies and different outcome variables, I ask two questions:

1. Using the maximum likelihood estimates from Tables 3, and assuming that the worker has hyperbolic preferences, what is the effect of the policy on the outcome?

2. How is the conclusion altered if one assumes instead that agents have exponential preferences?

To answer this second question, I re-estimate the model parameters under the restriction that $\beta=1$, and then evaluate the effects of the different policies. I call these calibrated policy evaluations. ${ }^{24}$

I evaluate the effect of any policy on four outcome variables: a) the expected duration of unemployment; b) the expected re-employment wage; c) the expected level of government payments; d) the individual's utility. With hyperbolic preferences, the correct notion of individual utility is difficult to define, because of the potential conflict between an individual's different selves: future events are discounted differently at different points in time, and an optimal strategy from today's perspective may no longer be optimal in the future. I follow here O'Donoghue and Rabin (2001), and evaluate the different policies using the perspective of the long-run self. The long-run self's utility is simply the utility derived from following the strategy chosen by the hyperbolic agent, discounted exponentially. Because of dynamic inconsistency, this strategy is not optimal from the long run self's perspective. The long run criterion can be thought of as

\footnotetext{
${ }^{24}$ One could also perform non-calibrated policy evaluations: holding all other parameters fixed, what is the effect of a given policy for different values of $\beta$ ? I omit these calculations beacuse as $\beta$ changes the benchmark values for the parameters of interest also vary substantially, making comparisons difficult.
} 
the utility criterion used by a voter who is not currently unemployed when deciding whether to implement a change to the UI system.

The five different policies are: a) cutting the level of unemployment benefits; b) a job search assistance program; c) monitoring the intensity of search effort; d) monitoring the job search acceptance strategy; e) a re-employment bonus program. I describe the policies below. The policies are modeled to resemble as closely as possible interventions that are, or have been, actually implemented. I restrict attention to the medium wage sample.

\subsection{Policies}

Benchmark. In the benchmark model, the unemployed worker receives Unemployment Insurance benefits for the first 26 weeks of the unemployment spell. The level of benefits for each population subgroup is taken as the average level of benefits for that group in the medium wage sample.

Cutting the level of unemployment benefits. This is the policy that is most commonly analyzed. I model it straightforwardly by cutting the level of unemployment benefits $b_{U I}$ by 20 percent.

Job search assistance program. Many government UI agencies attempt to improve claimants' re-employment prospects by providing a variety of job search assistance programs (see Meyer, 1995, for a survey). The program may include classroom training, help with writing resumes, facilitating contact between employers and job seekers, or one-on-one counseling sessions. I model these types of programs as a 10 percent reduction in search costs faced by the unemployed for the first 26 weeks of the unemployment spell. I assume that the government bears the burden of this cost reduction dollar for dollar. In terms of the model parameters, this means that the cost level parameter $k$ faced by the worker falls by 10 percent, and that government expenditures rise by $0.1 k$ for every week of insured unemployment.

Monitoring search intensity. Some UI systems combine the carrot represented by job search assistance programs, with the stick represented by a tightening of the eligibility requirements for receipt of benefits (see Meyer, 1995; Ashenfelter, Ashmore, and Deschênes, 1999; van den Berg, van der Klaauw and van Ours, 2004). This typically involves more frequent contacts with an employment agency representative to demonstrate active job seeking. I model this policy by assuming that the government can observe the level of search effort $s$ exerted by the worker: if this level falls below $\underline{s}=0.1$, the worker is no longer eligible to receive UI benefits. Monitoring the job acceptance strategy. In many UI systems, in order to be eligible 
for benefits, claimants must be actively looking for work, and must be available to start a job immediately. However, there might be some period at the beginning of a spell in which the claimant is allowed to restrict availability to jobs in his or her occupation or on the basis of pay. The following excerpt is taken from the United Kingdom's Jobseekers' Allowance Charter (www.employmentservice.gov.uk).

"If you are looking for work in your usual occupation, you may be allowed a period of between one and 13 weeks at the beginning of your claim, ... during which you are able to restrict your availability to that occupation and your normal rate of pay. You cannot normally refuse a job on the basis of pay after six months."

In modeling this policy, I assume that the government can observe the wage $w$ a worker is offered, and that the worker will lose eligibility to benefits if he or she rejects an offer below a certain threshold $w_{\min }$. I set $w_{\min }$ at 0 , meaning that any rejected offer will imply the loss of benefits.

Re-employment bonus. Several US states have experimented with re-employment bonus programs (Meyer, 1995), modeled on the successful Illinois Re-employment Bonus Experiment (Woodbury and Spiegelman, 1987). A typical re-employment bonus program could involve paying a bonus equal to 10 weeks of UI benefits to workers who found a job within 13 weeks, and then where able to hold that job for 13 more weeks. Translating this policy to our model is straightforward.

Figures $4 \mathrm{a}-4 \mathrm{e}$ present a graphical depiction of the effects of each policy.

\subsection{Results: calibrated policy evaluation}

I present the results of the calibrated policy evaluations in Table 7. The first four columns of the Table present the effects of the policies using parameter estimates from the hyperbolic model. These are simply the parameter estimates from Table 3. The last four columns present the effects of the policies using parameter estimates from a restricted model, where $\beta$ is set equal to 1 . For each policy, I report its estimated effect both in levels and in logs. The reported change in utility can be interpreted as the certainty equivalent of each policy: it is the lump sum amount that the worker would be willing to pay immediately in order to see the policy implemented (alternatively, it is the lump sum amount by which one would need to compensate the worker immediately in order to implement the policy).

A 20 percent decrease in the level of UI benefits leads to roughly a one week drop in expected 
duration in the hyperbolic model, and a 1.14 weeks drop in expected duration in the exponential model. This corresponds to a 4.36 to 5.13 percentage decrease in duration, or an elasticity of duration with respect to benefits between 0.22 and 0.26 . This estimate falls squarely in the range of previous findings in the literature (see Devine and Kiefer, 1991). The effect of the change in benefits on the expected re-employment wage is negligible: this appears to be true of all policies, and confirms that search effort is the main channel driving variation in exit rates. Expected government expenditures fall by more than 20 percent because of agents' behavioral response to the policy. The effect of the policy is similar for the hyperbolic and the exponential model in these dimensions. However, the utility drop for the hyperbolic agent is somewhat smaller than that for the exponential agent.

The job search assistance program is interesting in that it has a significantly larger effect on outcomes for the exponential agent than for the hyperbolic. The elasticity of expected duration with respect to search costs is 0.46 for the hyperbolic worker, but 0.65 for the exponential. Government expenditures rise by an order of magnitude: this seems to imply that a job search assistance program would always fail a cost-benefit analysis. This stands in contrast to the empirical findings on job search assistance programs: Meyer (1995) documents that four of the five job search assistance programs he analyzes yield higher benefits than costs to the government. $^{25}$ These differences can be reconciled if one believes that there are returns to scale in job search costs, so that the equivalent of one dollar in job search assistance to the individual worker actually costs less than one dollar to the government. Moreover, there might be other effects that are not taken into account in my simple formulation. For example, if the program shifts the distribution of potential wage offers, the cost-benefit analysis could be much more favorable.

The results of monitoring search intensity are striking. For the hyperbolic worker, this policy reduces expected unemployment duration by nearly eight weeks, and government expenditures (net of administrative costs) fall by 44 percent. Moreover, these drastic changes have actually a positive effect on the worker's utility! ${ }^{26}$ In contrast, the effect of this policy for the exponential worker is much smaller, and he would be willing to pay a substantial amount to avoid its implementation. These results were to be expected: the hyperbolic worker searches less than

\footnotetext{
${ }^{25}$ See Meyer (1995), Tables 6A and 6B.

${ }^{26}$ Here, worker type heterogeneity hides some important features of the policy. In the example, the restriction that search effort must be greater than 0.1 is binding only for the high cost types, and has no effect on the low cost types. Moreover the required level of search is low enough so that the high cost types prefer to search exactly 0.1 rather than lose their benefits. This puts them closer to the desired level of effort that would have been chosen by a time-consistent agent, so utility rises. A different constraint could actually lower utility.
} 
optimally from the long run self's perspective: he would be willing to pay for a commitment device that forces him to search more intensively in the future. The government's threat to cut benefits if he does not exert enough search effort acts exactly as this desirable commitment device. However, the magnitude of the effect is hardly plausible. In fact, Ashenfelter, Ashmore and Deschênes (1999) find no evidence that stricter enforcement and verification of work search behavior results in decreased UI claims and benefits. van den Berg, van der Klaauw and van Ours (2004) do find an effect of punitive sanctions on search effort for welfare recipients, but not nearly as large as that found here.

Monitoring the job acceptance strategy has a much smaller effect on all the outcome variables, for both the hyperbolic and the exponential models. Expected duration falls by around half a week in the hyperbolic model, and is essentially unchanged for the exponential. The expected re-employment wage decreases by at most 7 dollars. This finding confirms once more the relatively diminished importance of the reservation wage channel in determining the length of unemployment spells.

Finally, the re-employment bonus has quite a sizeable effect on all outcomes. Expected duration falls by more than two weeks for hyperbolic agents, and by two and a half weeks for exponential ones. These effects are somewhat higher than the effects found in the re-employment bonus experiments surveyed by Meyer. This could be due to the fact that the size of the bonus used here is relatively high compared to the actual bonus paid out in most of the experiments. The elasticity of duration with respect to the bonus is similar to that found in the Illinois claimant experiment (which was by far the most successful of the bonus experiments), and higher than that found in the other experiments.

In a separate analysis, not reported here, I found that most of the differences between the predicted effects of policies are due to differences in the remaining model parameters rather than in hyperbolic discounting per se. This does not mean that the hyperbolic discounting model should be dismissed because it does not deliver significantly different predictions from an exponential model. Quite the contrary: ignoring hyperbolic discounting leads one to estimate substantially different structural parameters, which in turn could lead one to draw incorrect conclusions on the effect of a given policy.

\section{Conclusion}

This paper estimates the structural parameters of a model of job search with hyperbolic discounting and endogenous search effort. The model is estimated using data on unemployment 
spells and accepted wages from the NLSY. The estimation strategy controls for both observed and unobserved heterogeneity.

The focus of the paper is the estimation of the time discounting parameters. Assuming that the parameters of the wage distribution, the cost function, and the value of time when unemployed are known, data on the duration of unemployment spells and accepted wages identify the time discounting parameters.

The model is estimated separately for three wage groups. In all three subsamples, and in particular for low and medium wage workers, the short run discounting parameter is smaller than one: this indicates a considerable amount of present bias. Likelihood ratio tests reject the exponential model. The model does a relatively good job at fitting observed outcomes such as the survivor function or the hazard rate, but it predicts an implausibly low conditional probability of acceptance. Imposing prior restrictions on quantities such as the acceptance probability or the cost of search may improve the fit of the model and may also help us gain precision in the estimation of poorly identified parameters.

The paper also uses the structural parameters to evaluate alternative policy interventions for the unemployed. The impact of different policies varies substantially depending on whether the model is calibrated with hyperbolic or exponential preferences.

In future work I plan to incorporate explicitly prior information on the structural parameters and on the unobserved outcomes of the job search process. Recent work by Lancaster (1997) and Koop and Poirier (2001) shows how Markov chain Monte Carlo simulation can be used to obtain posterior distributions for the parameters of interest in an optimal job search model. Koop and Poirier also argue that prior correlations between the parameters may aid in identification: intuitively, data information on the identified parameters "spills over" onto the unidentified parameters through the prior correlations. In our context, specifying a prior distribution on, say, the average probability of acceptance, provides exactly this type of prior correlation between the model parameters. Specifying prior distributions on the outcome variables has an additional advantage: it is probably much easier to formulate a sensible prior on the outcome variables than on some of the structural parameters. This is a promising avenue to follow if one wishes to improve the precision of the estimates of the discounting parameters. 


\section{A. Appendix A: Estimating the Marginal Cost of Search Elasticity}

Model. Consider an extension of the model described in the text in which workers have already chosen the optimal probability of receiving an offer $s$, and must now decide how to optimally allocate their time between alternative search methods. Assume that there are $K$ different methods that can be used to generate offers. Once the worker has chosen $s$, he must decide how intensively to use each method of search. Assume also that the effort exerted in this period has no bearing on the probability of receiving an offer in any of the latter periods, and that each search method generates offers from the same wage distribution. These assumptions make the model effectively static, and allow us to abstract from the issues of time discounting described in the text.

The worker's problem becomes one of optimally choosing intensity of search for each method so as to minimize search costs, subject to the constraint that the probability of receiving an offer be at least $s$. Formally, the problem is

$$
\begin{array}{ll} 
& \min _{X_{1}, \ldots, X_{K} ;} \sum_{j=1}^{K} c_{j} X_{j} \\
\text { s.t. } & : \quad P\left(X_{1}, \ldots, X_{K}\right) \geq s \\
X_{j} \quad \geq & 0, \quad j=1, \ldots, K
\end{array}
$$

This problem is very similar to that of a firm minimizing costs subject to a technological constraint, or to the dual problem of a worker who minimizes expenditure subject to a utility constraint. Letting $\lambda$ be the Lagrange multiplier, $(\lambda \geq 0)$, the first order conditions are:

$$
\begin{aligned}
-c_{j}+\lambda \frac{\partial P\left(X_{1}, \ldots, X_{K}\right)}{\partial X_{j}} & \leq 0, \quad \text { for } j=1, \ldots, K, \text { with equality when } X_{j}>0 \\
P\left(X_{1}, \ldots, X_{K}\right) & \geq s .
\end{aligned}
$$

These conditions give rise to the optimal intensities of search $X_{1}\left(c_{1}, \ldots, c_{K}, s\right), \ldots$, $X_{K}\left(c_{1}, \ldots, c_{K}, s\right)$, and to the minimized cost function $\tilde{c}\left(c_{1}, \ldots, c_{K}, s\right)$. In what follows I show how an appropriate choice of functional form can generate a simple tractable form for the cost function.

Analytical Solution. We make two important assumptions about the function $P(\cdot)$.

1. The probability of receiving an offer using method $j$ is independent of intensity of search in method $j^{\prime}, j^{\prime} \neq j$. 
2. The probability of receiving an offer using method $j, f\left(X_{j}\right)$, is an increasing and concave function of intensity of search in method $j$. Moreover, the probability of receiving an offer using method $j$ when one does not use the method at all is equal to zero. Therefore, $f^{\prime}(\cdot)>0, f^{\prime \prime}(\cdot)<0$, and $f(0)=0$.

We can then write

$$
P\left(X_{1}, \ldots, X_{K}\right)=1-\prod_{j=1}^{K}\left[1-f\left(X_{j}\right)\right] .
$$

This expression tells us that the probability of receiving an offer is equal to one minus the probability of receiving no offers at all using any of the $K$ methods.

Now let

$$
f\left(X_{j}\right)=1-\left(1+X_{j}\right)^{-\alpha_{j}}, \alpha_{j}>0 .
$$

It is easy to verify that this functional form satisfies all the necessary requirements. Using (A.1), we can express the first order conditions as

$$
\frac{c_{j}}{c_{k}}=\frac{f^{\prime}\left(X_{j}\right)}{f^{\prime}\left(X_{k}\right)} \frac{\left[1-f\left(X_{k}\right)\right]}{\left[1-f\left(X_{j}\right)\right]}
$$

and, using (A.2), we obtain

$$
\frac{c_{j}}{c_{k}}=\frac{\alpha_{j}}{\alpha_{k}} \frac{\left(1+X_{k}\right)}{\left(1+X_{j}\right)}
$$

which yields

$$
\left(1+X_{j}\right)=\frac{\alpha_{j}}{c_{j}} \frac{c_{k}}{\alpha_{k}}\left(1+X_{k}\right) .
$$

Now, using the fact that $s=1-\prod_{j=1}^{K}\left[1-f\left(X_{j}\right)\right]=1-\prod_{j=1}^{K}\left(1+X_{j}\right)^{-\alpha_{j}}$, we can solve for the individual $X_{j}$ 's:

$$
X_{j}(s)=\max \left\{\frac{\alpha_{j}}{c_{j}} \prod_{k=1}^{K}\left(\frac{c_{k}}{\alpha_{k}}\right)^{\alpha_{k} / \sum_{i} \alpha_{i}}(1-s)^{-1 / \sum_{i} \alpha_{i}}-1, \quad 0\right\}
$$

Then, the minimized cost function is

$$
\begin{aligned}
\tilde{c}(s) & =\sum_{j=1}^{K} c_{j} X_{j}(s) \\
& =\sum_{i=1}^{K}\left[\left(\sum_{J_{i}} \alpha_{j}\right)\left(\prod_{J_{i}}\left(\frac{c_{j}}{\alpha_{j}}\right)^{\sum_{J_{i}}^{\alpha_{j}}}\right)(1-s)^{-\frac{1}{\sum_{J_{i}} \alpha_{j}}}-\sum_{J_{i}} c_{j}\right] \times 1\left(s \in S_{i}\right)
\end{aligned}
$$

where $1(\cdot)$ is the standard indicator function; $S_{i}=\left\{s \mid \sum_{j=1}^{K} 1\left(X_{j}(s)>0\right)=i\right\}$ is the set of all possible $s$ values such that exactly $i$ methods of search are used; and $J_{i}=\left\{j: X_{j}(s)>0\right.$, for $\left.s \in S_{i}\right\}$ 
is the set of indicators for which search methods are used, when exactly $i$ methods are used. For large $s$, when all search methods are used, this function takes the form

$$
\tilde{c}(s)=A(1-s)^{-\varepsilon}-B .
$$

with $\varepsilon=1 / \sum_{j} \alpha_{j}$. The elasticity of the marginal cost of search is equal to $s(1+\varepsilon) /(1-s)$.

Estimation. We estimate the parameters of the cost function using the 1981 wave of the NLSY, that provides detailed information on the search activities of employed and unemployed youth. ${ }^{27}$ For those who searched for a job in the four weeks prior to the NLSY interview, we have information on whether search resulted in a job offer for each of eleven different methods. ${ }^{28}$ We also have information on the number of hours searched in the past week. I assume that the number of hours of search was constant in each of the past four weeks, and that the probability of receiving a job offer in a given week is independent of search effort in any other week. Let $X_{i j}$ be the number of hours spent searching by individual $i$ using method $j$, and let $O_{i j}$ be a dummy variable indicating whether method $j$ resulted in a job offer for individual $i$ in any of the past four weeks. The probability that individual $i$ received a job offer using method $j$ in any of the past four weeks is

$$
1-\left(1-f\left(X_{i j}\right)\right)^{4}=1-\left(1+X_{i j}\right)^{-4 \alpha_{j}}
$$

My estimation strategy consists of working with the conditional likelihood function:

$$
\begin{aligned}
\prod_{i=1}^{N} f\left(O_{i j} \mid X_{i j}, \alpha\right) & =\prod_{i=1}^{N} \prod_{j=1}^{K} f\left(O_{i j} \mid X_{i j}, \alpha_{j}\right) \\
& =\prod_{i=1}^{N} \prod_{j=1}^{K}\left[1-\left(1+X_{i j}\right)^{-4 \alpha_{j}}\right]^{O_{i j}}\left[\left(1+X_{i j}\right)^{-4 a_{j}}\right]^{1-O_{i j}}
\end{aligned}
$$

Note that each $\alpha$ can be estimated consistently by maximizing the likelihood separately for each search method. This simplifies the calculations considerably. Given $\alpha$, one can back out the cost coefficients $c_{1}, \ldots, c_{K-1}$ ( $c_{K}$ is normalized to 1$)$ from the first order conditions (A.3):

$$
\hat{c}_{j}=\frac{\hat{\alpha}_{j}}{\hat{\alpha}_{K}} \frac{\left(1+\bar{x}_{K}\right)}{\left(1+\bar{x}_{j}\right)}
$$

\footnotetext{
${ }^{27}$ This is the same data used in Holzer (1988).

${ }^{28}$ These were: 1) checked with the state employment agency; 2) checked with a private employment agency; 3 ) asked friends and relatives about jobs; 4) placed or answered newspaper ads; 5) took the civil servces test or applied for a government job; 6) contacted any public organization; 7) contacted a school placement office; 8) asked teachers or professors about jobs; 9) checked with a labor union; 10) checked directly with employers; 11) other methods.
} 
The estimation is carried out on the subsample of youth who were searching while unemployed, and did not list being in school as their main activity during the survey week. I focus on the four most popular search methods (state employment service, friends and relatives, newspaper ads, direct employer contact), and aggregate the other methods into the "other methods" category.

The first three columns of Appendix Table 1 present the percentage of the sample using each method, and, conditional on using the method, the average hours spent searching and the probability of receiving an offer in any of the past four weeks. I also report the implied weekly probability of receiving an offer. The next two columns report the estimates and standard errors for the parameters of the probability function, $\alpha_{1}, \ldots, \alpha_{5}$, and for the cost coefficients $c_{1}, \ldots, c_{5}$.

Given $\alpha$ and $c$, one can evaluate the cost function $\tilde{c}(s)$ at various levels of search intensity $s$. These estimated values are then used to estimate the parameters of the constant marginal cost elasticity cost function, $c(s)=k s^{1+\eta}$, used in the dynamic programming model. The estimation is performed by running a weighted least squares regression of $\log \tilde{c}(s)$ on $\log s$, where the weights are proportional to a normal density centered at the estimated mean probability of receiving a job offer. ${ }^{29}$ The results of this regression are

$$
\begin{aligned}
\log \tilde{c}(s)= & 4.412+1.408 \log s \\
& (0.003)(0.0011)
\end{aligned}
$$

The estimated constant marginal cost elasticity $\eta$ is then equal to the coefficient on $\log s$ minus one. This gives rise to the benchmark value of $\eta=0.4$ used in the text.

\footnotetext{
${ }^{29}$ The regression is weighted because the actual cost function $\tilde{c}(s)$ and the approximate cost function $c(s)$ can differ substantially at extreme values of $s$. Therefore we give more weight to those values of $s$ that have higher density empirically.
} 


\section{B. Appendix B: Calculation of Predicted Values}

Let us first define the aggregate exit rate and the aggregate survivor function. Let $h_{j t}=$ $s_{j t}\left[1-F\left(w_{j t}^{*}\right)\right]$ be the exit rate for a worker of type $j(j=H I, L O)$ in week $t$ of an unemployment spell. The exit rate is the product of the probability of receiving an offer $s_{j t}$ and the probability of accepting the offer $\left[1-F\left(w_{j t}^{*}\right)\right]$. Let $D$ be the random variable indicating the length of the unemployment spell. The survivor function at week $t$ is the probability that a spell lasts $t$ weeks or longer. The survivor function at week $t$ for type $j$ is

$$
P(D \geq t \mid j)=z_{j t}=\prod_{s=1}^{t-1}\left(1-h_{j t}\right),
$$

with the convention that $h_{j 0}=0$. Let $\phi_{j 1}$ be the proportion of workers of type $j$ in the sample, and let $\phi_{j t}$ be the proportion of workers of type $j$ who are still unemployed at time $t$ :

$$
\begin{aligned}
\phi_{j t} & =P(j \mid D \geq t)=\frac{P(D \geq t \mid j) P(j)}{\sum_{k} P(D \geq t \mid k) P(k)} \\
& =\frac{\phi_{j 1} z_{j t}}{\sum_{k} \phi_{k 1} z_{k t}} .
\end{aligned}
$$

The aggregate exit rate in week $t$ is $H_{t}$ :

$$
\begin{aligned}
H_{t} & =P(D=t \mid D \geq t)=\sum_{j} P(D=t \mid D \geq t, j) P(j \mid D \geq t) \\
& =\sum_{j} h_{j t} \phi_{j t} .
\end{aligned}
$$

The aggregate survivor function in week $t$ is $Z_{t}$ :

$$
Z_{t}=P(D \geq t)=\prod_{s=1}^{t-1}\left(1-H_{s}\right) .
$$

Expected Duration. Aggregate expected duration is given by:

$$
\begin{aligned}
E(D) & =\sum_{t=1}^{\infty} t P(D=t)=\sum_{t=1}^{\infty} t P(D=t \mid D \geq t) P(D \geq t) \\
& =\sum_{t=1}^{\infty} t H_{t} Z_{t} .30
\end{aligned}
$$

Expected Re-employment Wage. Let $\tilde{w}_{j t}$ be the expected re-employment wage for workers of type $j$ who exit unemployment in week $t$. The aggregate expected re-employment wage in 
week $t$ is given by:

$$
\begin{aligned}
\tilde{W}_{t} & =E(\tilde{W} \mid D=t)=\sum_{j} E(\tilde{W} \mid D=t, j) P(j \mid D=t) \\
& =\sum_{j} \tilde{w}_{j t} \frac{P(D=t \mid j) P(j)}{\sum_{k} P(D=t \mid k) P(k)}=\sum_{j} \tilde{w}_{j t} \frac{z_{j t} h_{j t} \phi_{j 1}}{\sum_{k} z_{k t} h_{k t} \phi_{k 1}} \\
& =\sum_{j} \tilde{w}_{j t} \frac{h_{j t} \phi_{j t} \sum_{k} \phi_{k 1} z_{k t}}{\sum_{k} h_{k t} \phi_{k t} \sum_{k} \phi_{k 1} z_{k t}}=\frac{\sum_{j} \tilde{w}_{j t} h_{j t} \phi_{j t}}{H_{t}}
\end{aligned}
$$

where the second to last equality is derived by using (B.1) in both the numerator and the denominator. The ex-ante expected re-employment wage is then

$$
E(\tilde{W})=\sum_{t=1}^{\infty} \tilde{W}_{t} H_{t} Z_{t}
$$

Average weekly cost of search, long run utility. The predicted values for the expectation of the average weekly cost of search and of long run utility are derived in analogous manner to the expected re-employment wage. Let $\bar{c}_{j t}=t^{-1} \sum_{s=1}^{t} c\left(s_{j t}\right)$ be the average weekly cost of search for a worker of type $j$ who exits unemployment in week $t$. Let $u_{j t}$ be the expected long run utility for a worker of type $j$ who exits unemployment at time $t$. Then, using a similar line of argument as for the calculation of the expected re-employment wage, we obtain the expected aggregate cost of search

$$
E(\bar{C})=\sum_{t=1}^{\infty} \bar{C}_{t} H_{t} Z_{t}=\sum_{t=1}^{\infty} \sum_{j} \frac{\bar{c}_{j t} h_{j t} \phi_{j t}}{H_{t}},
$$

and the expected aggregate utility

$$
E(U)=\sum_{t=1}^{\infty} U_{t} H_{t} Z_{t}=\sum_{t=1}^{\infty} \sum_{j} \frac{u_{j t} h_{j t} \phi_{j t}}{H_{t}} .
$$

Conditional Probability of Acceptance. The conditional probability of acceptance is calculated as the expected total number of accepted offers divided by the expected total number of offers. Since eventually all workers exit unemployment, the expected total number of accepted offers is equal to 1 . The expected total number of offers is the sum, over all periods $t$, of the probability of receiving an offer conditional on being unemployed at $t$, times the probability of being unemployed at $t$. Let $S_{t}=\sum_{j} \phi_{j t} s_{j t}$ be the aggregate expected probability of receiving an offer in period $t$. Then, the conditional probability of acceptance is:

$$
\operatorname{Pr}(\text { Acceptance } \mid \text { Offer })=\frac{\sum_{t=1}^{\infty} H_{t} Z_{t}}{\sum_{t=1}^{\infty} S_{t} Z_{t}}=\frac{1}{\sum_{t=1}^{\infty} S_{t} Z_{t}} .
$$




\section{References}

[1] Ainslie, George W. Picoeconomics. Cambridge: Cambridge University Press, 1992.

[2] Angeletos, George-Marios; Laibson, David I.; Repetto, Andrea; Tobacman, Jeremy and Weinberg, Stephen. "The Hyperbolic Buffer Stock Model: Calibration, Simulation, and Empirical Evaluation." Journal of Economic Perspectives, Summer 2001, 15(3), pp. 47-68.

[3] Ashenfelter, Orley; Ashmore, David and Deschênes, Olivier. "Do Unemployment Insurance Recipients Actively Seek Work? Randomized Trials in Four U.S. States." National Bureau of Economic Research (Cambridge, MA) Working Paper No. W6982, February 1999.

[4] Benzion, Uri; Rapoport, Amnon and Yagil, Joseph. "Discount Rates Inferred from Decisions: an Experimental Study." Management Science, March 1989, 35(3), pp. 270-84.

[5] Blau, David M. and Robins, Philip K. "Job Search Outcomes for the Employed and Unemployed." Journal of Political Economy, June 1990, 98(3), pp. 637-55.

[6] Burdett, Kenneth and Ondrich, Jan I. "How Changes in Labor Demand Affect Unemployed Workers." Journal of Labor Economics, January 1985, 3(1), pp. 1-10.

[7] DellaVigna, Stefano and Paserman, M. Daniele. "Job Search and Hyperbolic Discounting." The Maurice Falk Institute for Economic Research in Israel (Jerusalem), Discussion Paper No. 00.15, December 2000.

[8] Devine, Theresa J. and Kiefer, Nicholas M. Empirical Labor Economics: The Search Approach. New York and Oxford: Oxford University Press, 1991.

[9] Diamond, Peter and Köszegi, Botond. "Quasi-Hyperbolic Discounting and Retirement." Journal of Public Economics, 2003, forthcoming.

[10] Fang, Hanming and Silverman, Dan. "Time-inconsistency and Welfare Program Participation: Evidence from the NLSY." Mimeo., Yale University, April 2002.

[11] Gruber, Jonathan and Köszegi, Botond. "Is Addiction 'Rational'? Theory and Evidence." Quarterly Journal of Economics, November 2001, 116(4), pp. 1261-1304.

[12] Harris, Christopher, and Laibson, David I. "Dynamic Choices of Hyperbolic Consumers." Econometrica, July 2001, 69(4), pp. 935-957. 
[13] Holzer, Harry J. "Search Method Use by Unemployed Youth." Journal of Labor Economics, January 1988, 6(1), pp. 1-20.

[14] Kirby, Kris N. "Bidding on the Future: Evidence Against Normative Discounting of Delayed Rewards." Journal of Experimental Psychology: General, March 1997, 126(1), pp. $54-70$.

[15] Kirby, Kris N. and Herrnstein, R.J. "Preference Reversals Due to Myopic Discounting of Delayed Reward." Psychological Science, March 1995, 6(2), pp. 83-89.

[16] Koop, Gary and Poirier, Dale J. "Testing for Optimality in Job Search Models." Econometrics Journal, December 2001, 4(2), pp. 257-272.

[17] Laibson, David I. "Golden Eggs and Hyperbolic Discounting." Quarterly Journal of Economics, May 1997, 112(2), pp. 443-77.

[18] Laibson, David I.; Repetto, Andrea and Tobacman, Jeremy. "Self Control and Saving for Retirement." Brookings Papers on Economic Activity, 1998, 0(1), pp. 91-172.

[19] Laibson, David I.; Repetto, Andrea and Tobacman, Jeremy. "A Debt Puzzle," in Knowledge, Information and Expectations in Modern Economics: In Honor of edmund S. Phelps, Philippe Aghion, Roman Frydman, Joseph Stiglitz and Michael Woodford eds., Princeton: Princeton University Press, 2003.

[20] Lancaster, Tony. "Exact Structural Inference in Optimal Job-Search Models." Journal of Business and Economic Statistics, April 1997, 15(2), pp. 165-179.

[21] Lippman, Steven A. and McCall, John J. "The Economics of Job Search: A Survey." Economic Inquiry, September 1976, 14 (1), pp. 155-189.

[22] Meyer, Bruce D. "Lessons From the U.S. Unemployment Insurance Experiments." Journal of Economic Literature, March 1995, 33(1), pp. 91-131.

[23] O'Donoghue, Ted D. and Rabin, Matthew. "Doing It Now or Later." American Economic Review, March 1999, 89(1), pp. 103-124.

[24] O'Donoghue, Ted D. and Rabin, Matthew. "Choice and Procrastination." Quarterly Journal of Economics, February 2001, 126(1), pp. 121-160.

[25] Oswald, Andrew J. "Happiness and Economic Performance." Economic Journal, November 1997, 107(445), pp. 1815-1831. 
[26] Phelps, Edmund S. and Pollak R.A. "On Second-Best National Saving and GameEquilibrium Growth." Review of Economic Studies, April 1968, 35(2), pp. 85-199.

[27] Thaler, Richard H. "Some Empirical Evidence on Dynamic Inconsistency." Economics Letters, 1981, 8, pp. 201-207.

[28] van den Berg, Gerard J. "Nonstationarity in Job Search Theory." Review of Economic Studies, April 1990, 57(2), pp. 255-277.

[29] van den Berg, Gerard J.; van der Klaauw, Bas and van Ours, Jan C. "Punitive Sanctions and the Transition Rate from Welfare to Work." Journal of Labor Economics, 2004, forthcoming.

[30] Winkelmann, L. and Winkelmann, R. "Why are the Unemployed so Unhappy? Evidence from Panel Data." Economica, February 1998, 65(257), pp. 1-15.

[31] Wolpin, Kenneth I. "Estimating a Structural Search Model: The Transition from School to Work." Econometrica, July 1987, 55(4), pp. 801-817.

[32] Woodbury, Stephen A. and Spiegelman, Robert G. "Bonuses to Workers and Employers to Reduce Unemployment: Randomized Trials in Illinois." American Economic Review, September 1987, 77 (4), pp. 513-530. 


\section{Table 1: Summary of Theoretical Results ${ }^{\dagger}$}

\begin{tabular}{lccccc} 
Variable Description & Parameter & $\begin{array}{c}\text { Search } \\
\text { Effort }\end{array}$ & $\begin{array}{c}\text { Reservation } \\
\text { Wage }\end{array}$ & $\begin{array}{c}\text { Probability } \\
\text { Of Acceptance }\end{array}$ & Exit rate \\
\hline Short Run Impatience & $\beta$ & + & $+/=^{1}$ & $-/=^{2}$ & + \\
Long Run Impatience & $\delta$ & + & + & - & $-{ }^{3}$ \\
Utility of Leisure & $\mathrm{b}$ & - & + & - & - \\
$\begin{array}{l}\text { Location of wage } \\
\text { distribution }\end{array}$ & $\mu$ & + & + & + & + \\
$\begin{array}{l}\text { Dispersion of wage } \\
\text { distribution }\end{array}$ & $\sigma$ & + & + & + & $?$ \\
$\begin{array}{l}\text { Probability of layoff } \\
\text { Cost of search }\end{array}$ & $q$ & - & - & + & $?$ \\
\hline
\end{tabular}

\footnotetext{
$\dagger$ Notes: The entries in the Table represent the partial effects of a change in the parameters on job search outcomes.

1 The effect of a decrease in $\beta$ on the reservation wage is negative for sophisticated workers, and null for naive workers.

2 The effect of a decrease in $\beta$ on the probability of acceptance is positive for sophisticated workers, and null for naive workers.

${ }^{3}$ Extensive simulations show that the sign of the partial derivative of the exit rate with respect to $\delta$ is negative. See text for details.
} 


\section{Table 2: Summary Statistics $^{\dagger}$}

\begin{tabular}{|c|c|c|c|c|c|c|}
\hline \multirow[b]{3}{*}{ Duration } & \multicolumn{2}{|c|}{ Low wage } & \multicolumn{2}{|c|}{ Medium Wage } & \multicolumn{2}{|c|}{ High Wage } \\
\hline & Mean & Std. Dev. & Mean & Std. Dev. & Mean. & Std. Dev. \\
\hline & 24.81 & 27.04 & 21.57 & 24.60 & 16.47 & 18.67 \\
\hline $\begin{array}{l}\text { Re-employment } \\
\text { wage }\end{array}$ & 180.25 & 89.09 & 265.90 & 104.17 & 462.34 & 201.55 \\
\hline $\begin{array}{l}\text { Log }(\mathrm{Re}- \\
\text { employment wage) }\end{array}$ & 5.10 & 0.43 & 5.51 & 0.41 & 6.04 & 0.46 \\
\hline Previous Wage & 150.10 & 37.31 & 277.27 & 50.05 & 532.48 & 204.98 \\
\hline UI benefits & 95.39 & 42.53 & 138.52 & 94.71 & 167.81 & 47.70 \\
\hline Replacement rate & 0.72 & 0.65 & 0.51 & 0.37 & 0.34 & 0.12 \\
\hline Age & 28.18 & 4.13 & 29.07 & 3.89 & 30.49 & 3.90 \\
\hline Education & 11.67 & 1.83 & 11.90 & 1.70 & 12.61 & 1.83 \\
\hline Married & 0.33 & 0.47 & 0.44 & 0.50 & 0.57 & 0.50 \\
\hline AFQT & 36.13 & 25.92 & 47.22 & 26.82 & 55.72 & 25.10 \\
\hline $\begin{array}{l}\text { Number of } \\
\text { observations }\end{array}$ & & & & & & \\
\hline
\end{tabular}

\footnotetext{
$\dagger$ Notes: The sample includes all spells of unemployment for males not enrolled in school and not in the military, reported after 1985, in which Unemployment Insurance benefits were received for at least one week.

Source: Author's calculations from the NLSY.
} 


\section{Table 3: Estimated Model Parameters}

\begin{tabular}{|c|c|c|c|c|c|c|}
\hline \multirow{2}{*}{ Discounting Parameters } & \multicolumn{2}{|c|}{$\begin{array}{l}\text { Low Wage } \\
\text { Sample }\end{array}$} & \multicolumn{2}{|c|}{$\begin{array}{l}\text { Medium Wage } \\
\text { Sample }\end{array}$} & \multicolumn{2}{|c|}{$\begin{array}{l}\text { High Wage } \\
\text { Sample }\end{array}$} \\
\hline & Coeff. & Std. Err. & Coeff. & Std. Err. & Coeff. & Std. Err. \\
\hline Discounting Parameters & 0.4021 & $(0.1075)$ & 0.4833 & $(0.1971)$ & 0.8937 & $(0.1441)$ \\
\hline 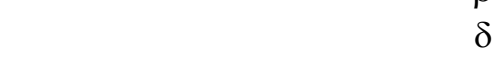 & 0.9962 & $(0.1848)$ & $1.0000^{*}$ & $(0.0001)$ & 0.9989 & $(0.1798)$ \\
\hline \multicolumn{7}{|l|}{$\begin{array}{l}\text { Value of time when } \\
\text { unemployed }\end{array}$} \\
\hline 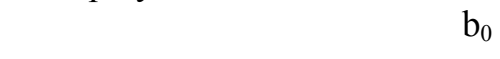 & -141.61 & $(61.16)$ & -164.31 & $(61.43)$ & -308.78 & $(193.53)$ \\
\hline \multicolumn{7}{|l|}{$\begin{array}{l}\text { Parameters of the wage offer } \\
\text { distribution }\end{array}$} \\
\hline$\mu_{1}$ (unmarried, low AFQT) & 4.1545 & $(0.1996)$ & 5.0230 & $(0.1547)$ & 5.4296 & $(0.3408)$ \\
\hline$\mu_{2}$ (unmarried, medium AFQT) & 4.3613 & $(0.1789)$ & 5.0993 & $(0.1230)$ & 5.2297 & $(0.1331)$ \\
\hline$\mu_{3}$ (unmarried, high AFQT) & 4.9503 & $(0.2341)$ & 4.8658 & $(0.1425)$ & 4.5543 & $(0.1785)$ \\
\hline$\mu_{4}$ (married, low AFQT) & 4.3954 & $(0.2187)$ & 5.1694 & $(0.1192)$ & 5.6692 & $(0.1956)$ \\
\hline$\mu_{5}($ married, medium AFQT) & 4.2771 & $(0.1337)$ & 5.2790 & $(0.1150)$ & 5.4365 & $(0.1361)$ \\
\hline$\mu_{6}$ (married, high AFQT) & 4.1175 & $(0.2683)$ & 5.0735 & $(0.0837)$ & 5.3669 & $(0.1235)$ \\
\hline$\sigma_{1}$ (unmarried, low AFQT) & 0.7196 & $(0.0723)$ & 0.5614 & $(0.0585)$ & 0.7444 & $(0.1659)$ \\
\hline$\sigma_{2}$ (unmarried, medium AFQT) & 0.5760 & $(0.0516)$ & 0.4122 & $(0.0453)$ & 0.5810 & $(0.0502)$ \\
\hline$\sigma_{3}$ (unmarried, high AFQT) & 0.2786 & $(0.2653)$ & 0.4793 & $(0.0397)$ & 0.7108 & $(0.0944)$ \\
\hline$\sigma_{4}$ (married, low AFQT) & 0.5413 & $(0.0770)$ & 0.3525 & $(0.0459)$ & 0.5658 & $(0.0767)$ \\
\hline$\sigma_{5}($ married, medium AFQT) & 0.5187 & $(0.0423)$ & 0.3068 & $(0.0410)$ & 0.4649 & $(0.0424)$ \\
\hline$\sigma_{6}($ married, high AFQT) & 0.5715 & $(0.1039)$ & 0.3463 & $(0.0320)$ & 0.5503 & $(0.0436)$ \\
\hline
\end{tabular}

Standard Deviation of the

Measurement Error in Log

Wages

$$
\begin{array}{lllllll}
\sigma_{\mathrm{u}} & 0.1330 & (0.0256) & 0.2256 & (0.0197) & 0.0990 & (0.0182)
\end{array}
$$

Parameters of the cost of search function

$\begin{array}{rcccccc}\mathrm{k}_{1} \text { (unmarried, low AFQT) } & 678 & (382) & 2,998 & (1,818) & 9,085 & (8,106) \\ \mathrm{k}_{2}(\text { unmarried, medium AFQT) } & 359 & (217) & 1,174 & (797) & 1,198 & (879) \\ \mathrm{k}_{3} \text { (unmarried, high AFQT) } & 3,362 & (3,442) & 325 & (273) & 304 & (194) \\ \mathrm{k}_{4} \text { (married, low AFQT) } & 451 & (343) & 1,002 & (780) & 8,499 & (6,681) \\ \mathrm{k}_{5}(\text { married, medium AFQT) } & 83 & (31) & 905 & (667) & 1,216 & (873) \\ \mathrm{k}_{6} \text { (married, high AFQT) } & 118 & (160) & 170 & (98) & 1,073 & (685)\end{array}$

\footnotetext{
Notes: Standard errors in parentheses. Standard errors were obtained using the outer product gradient method.

* : Indicates that the estimate is at the border of the parameter space: standard errors should be viewed with caution.

Source: Author's calculations from the NLSY.
} 


\section{$\underline{\text { Table 3: Estimated Model Parameters - Continued }}{ }^{\dagger}$}

\begin{tabular}{|c|c|c|c|c|c|c|}
\hline \multirow{2}{*}{$\begin{array}{l}\text { Parameters of the } \\
\text { heterogeneity } \\
\text { distribution }\end{array}$} & \multicolumn{2}{|c|}{ Low Wage Sample } & \multicolumn{2}{|c|}{$\begin{array}{c}\text { Medium Wage } \\
\text { Sample }\end{array}$} & \multicolumn{2}{|c|}{ High Wage Sample } \\
\hline & Coeff. & Std. Err. & Coeff. & Std. Err. & Coeff. & Std. Err. \\
\hline $\begin{array}{r}\Delta \mu \\
\Delta \mathrm{k} \\
\mathrm{p}\end{array}$ & $\begin{array}{l}0.5456 \\
12,374 \\
0.2962\end{array}$ & $\begin{array}{c}(0.1262) \\
(4,217) \\
(0.0505)\end{array}$ & $\begin{array}{l}0.0201 \\
19,114 \\
0.2276\end{array}$ & $\begin{array}{c}(0.1185) \\
(7,675) \\
(0.0330)\end{array}$ & $\begin{array}{l}0.2179 \\
56,857 \\
0.2974\end{array}$ & $\begin{array}{l}(0.1412) \\
(28,021) \\
(0.0341)\end{array}$ \\
\hline $\begin{array}{r}\text { Number of } \\
\text { Observations }\end{array}$ & \multicolumn{2}{|c|}{450} & \multicolumn{2}{|c|}{898} & \multicolumn{2}{|c|}{449} \\
\hline Log-Likelihood & \multicolumn{2}{|c|}{-4041.5} & \multicolumn{2}{|c|}{-8484.8} & \multicolumn{2}{|c|}{-4270.2} \\
\hline LR statistic for $\beta=1$ & \multicolumn{2}{|c|}{17.2268} & \multicolumn{2}{|c|}{13.2375} & \multicolumn{2}{|c|}{3.0826} \\
\hline p-value ${ }^{1}$ & \multicolumn{2}{|c|}{0.00} & \multicolumn{2}{|c|}{0.00} & \multicolumn{2}{|c|}{0.08} \\
\hline
\end{tabular}

† Notes: Standard errors in parentheses. Standard errors were obtained using the outer product gradient method.

* : Indicates that the estimate is at the border of the parameter space: standard errors should be viewed with caution.

Source: Author's calculations from the NLSY.

${ }^{1}$ Based on a $\chi^{2}(1)$ distribution. 


\section{Table 4: Alternative Forms of Hetrogeneity}

\begin{tabular}{|c|c|c|c|}
\hline & Alternative 1 & Alternative 2 & Alternative 3 \\
\hline $\begin{array}{l}\text { Observed Heterogeneity } \\
\text { Variables }\end{array}$ & $\begin{array}{l}\text { AFQT, marital } \\
\text { status }\end{array}$ & $\begin{array}{l}\text { AFQT, marital } \\
\text { status, race }\end{array}$ & $\begin{array}{l}\mathrm{AFQT}, \underset{\text { mace }}{\text { matal status, }} \\
\text {, }\end{array}$ \\
\hline $\begin{array}{l}\text { Number of mass points in } \\
\text { unobserved heterogeneity } \\
\text { distribution }\end{array}$ & 3 & 2 & 2 \\
\hline $\begin{array}{l}\text { Link between unobserved } \\
\text { heterogeneity and control } \\
\text { variables }\end{array}$ & Independent & Independent & $P(i$ is type $j)=\frac{\mathrm{e}^{\alpha_{k}{ }^{\prime} \mathrm{X}_{\mathrm{i}}}}{\sum_{k} e^{\alpha_{k} X_{i}}}$ \\
\hline$\beta$ & 0.455 & 0.464 & 0.697 \\
\hline s.e. of $\beta$ & $(0.142)$ & $(0.051)$ & $(0.190)$ \\
\hline Log-Likelihood & -8478.6 & -8453.1 & -8452.8 \\
\hline $\begin{array}{l}\text { LR statistic for the } \\
\text { restrictions imposed in } \\
\text { Table } 3\end{array}$ & 11.6 & 63.4 & - \\
\hline$p$-value & 0.003 & 0.000 & - \\
\hline LR statistic for $\beta=1$ & 1.45 & 6.04 & 0.97 \\
\hline p-value & 0.23 & 0.01 & 0.33 \\
\hline
\end{tabular}




\section{Table 5: Predicted Outcomes - Averages ${ }^{\dagger}$}

$\underline{\text { LOW WAGE }}$

Duration

Re-employment

wage

\begin{tabular}{cc} 
Empirical & Predicted \\
\hline 24.81 & 26.06 \\
180.25 & 178.82 \\
5.10 & 5.18 \\
- & 0.20 \\
- & 70.73
\end{tabular}

\section{MEDIUM WAGE}

Duration

\begin{tabular}{cc} 
Empirical & Predicted \\
\hline 21.57 & 22.43 \\
265.90 & 259.35
\end{tabular}

wage

Log (re-employment

wage)

5.51

5.55

Conditional

acceptance

probability

Cost of search ${ }^{1}$

121.89

\section{$\underline{\text { HIGH WAGE }}$}

Duration

\begin{tabular}{cc} 
Empirical & Predicted \\
\hline 16.47 & 17.84 \\
462.34 & 455.01 \\
6.04 & 6.11 \\
- & 0.14 \\
- & 760.92
\end{tabular}

Re-employment

wage

Log (re-employment

wage)

Conditional

acceptance

760.92

\footnotetext{
$\dagger$ Notes: Entries in the Table represent the ex-ante expected values of the relevant variables. Source: Author's calculations from the NLSY.

${ }^{1}$ The cost of search is the average weekly cost of search over the unemployment spell.
} 


\section{Table 6: Predicted Outcomes: Dynamics ${ }^{\dagger}$}

Low Wage

\begin{tabular}{lccccccc} 
& $\begin{array}{c}\text { Empirical } \\
\text { survivor } \\
\text { function }\end{array}$ & $\begin{array}{c}\text { Predicted } \\
\text { survivor }\end{array}$ & Exit rate & $\begin{array}{c}\text { Offer } \\
\text { Probability }\end{array}$ & $\begin{array}{c}\text { Conditional } \\
\text { acceptance } \\
\text { probability }\end{array}$ & $\begin{array}{c}\text { Expected re- } \\
\text { employment } \\
\text { wage }\end{array}$ & $\begin{array}{c}\text { Cost of } \\
\text { search }\end{array}$ \\
\cline { 2 - 8 } Week 1 & 1.00 & 1.00 & 0.040 & 0.263 & 0.152 & 186.37 & 63.36 \\
Week 13 & 0.60 & 0.62 & 0.039 & 0.226 & 0.173 & 183.44 & 66.75 \\
Week 26 & 0.32 & 0.36 & 0.047 & 0.212 & 0.224 & 175.74 & 88.48 \\
Week 52 & 0.14 & 0.12 & 0.034 & 0.099 & 0.344 & 169.59 & 80.41 \\
Week 104 & 0.03 & 0.03 & 0.025 & 0.033 & 0.770 & 161.32 & 74.48
\end{tabular}

Medium Wage

\begin{tabular}{lccccccc} 
& $\begin{array}{c}\text { Empirical } \\
\text { survivor } \\
\text { function }\end{array}$ & $\begin{array}{c}\text { Predicted } \\
\text { survivor }\end{array}$ & Exit rate & $\begin{array}{c}\text { Offer } \\
\text { Probability }\end{array}$ & $\begin{array}{c}\text { Conditional } \\
\text { acceptance } \\
\text { probability }\end{array}$ & $\begin{array}{c}\text { Expected re- } \\
\text { employment } \\
\text { wage }\end{array}$ & $\begin{array}{c}\text { Cost of } \\
\text { search }\end{array}$ \\
\cline { 2 - 7 } Week 1 & 1.00 & 1.00 & 0.051 & 0.246 & 0.207 & 277.67 & 111.07 \\
Week 13 & 0.56 & 0.55 & 0.047 & 0.202 & 0.232 & 270.18 & 112.72 \\
Week 26 & 0.27 & 0.29 & 0.055 & 0.186 & 0.296 & 255.87 & 151.00 \\
Week 52 & 0.10 & 0.09 & 0.033 & 0.067 & 0.493 & 220.87 & 124.18 \\
Week 104 & 0.03 & 0.02 & 0.025 & 0.028 & 0.901 & 188.41 & 111.18
\end{tabular}

High Wage

\begin{tabular}{lccccccc} 
& $\begin{array}{c}\text { Empirical } \\
\text { survivor } \\
\text { function }\end{array}$ & $\begin{array}{c}\text { Predicted } \\
\text { survivor }\end{array}$ & Exit rate & $\begin{array}{c}\text { Offer } \\
\text { Probability }\end{array}$ & $\begin{array}{c}\text { Conditional } \\
\text { acceptance } \\
\text { probability }\end{array}$ & $\begin{array}{c}\text { Expected re- } \\
\text { employment } \\
\text { wage }\end{array}$ & $\begin{array}{c}\text { Cost of } \\
\text { search }\end{array}$ \\
\cline { 2 - 8 } Week 1 & 1.00 & 1.00 & 0.070 & 0.562 & 0.125 & 496.16 & 732.72 \\
Week 13 & 0.47 & 0.45 & 0.060 & 0.434 & 0.137 & 470.31 & 681.07 \\
Week 26 & 0.19 & 0.21 & 0.054 & 0.323 & 0.168 & 427.92 & 700.25 \\
Week 52 & 0.05 & 0.06 & 0.038 & 0.167 & 0.225 & 352.03 & 543.47 \\
Week 104 & 0.01 & 0.01 & 0.026 & 0.069 & 0.377 & 272.42 & 367.55
\end{tabular}

\footnotetext{
† Source: Author’s calculations.
} 


\title{
Table 7: Policy Evaluations $^{\dagger}$
}

\author{
Medium Wage Sample
}

Unrestricted Model -

\section{Hyperbolic}

\begin{tabular}{cccc}
$\begin{array}{c}\text { Expected } \\
\text { Duration } \\
\text { (weeks) }\end{array}$ & Expected & Gov't & Long- \\
Expenditure & Run \\
& & $(\$)$ & Utility \\
\hline
\end{tabular}

Benchmark

$22.43 \quad 259.36 \quad 2052$

\section{Change in UI}

benefits
In levels

in $\operatorname{logs}(\times 100)$

$-0.83$

$-0.32$

$-480$

$-26.70$

$-245$

$-4.36$

\section{Job search}

assistance

in level

in $\operatorname{logs}(\times 100)$

$\begin{array}{rrrr}-1.02 & 2.66 & 10,630 & 502 \\ -4.65 & 1.02 & 182.15\end{array}$

$\begin{array}{lll}-4.65 & 1.02 & 182.15\end{array}$

\section{Monitoring}

\section{search effort}

\begin{tabular}{|c|c|c|c|}
\hline & -7.69 & 0.93 & -360 \\
\hline$n \operatorname{logs}(x$ & -41.96 & -0.20 & -44.72 \\
\hline
\end{tabular}

\section{Monitoring}

acceptance

\section{in levels \\ in $\operatorname{logs}(\times 100)$ \\ Employment \\ bonus}

$-4.52$

$-1.76$

$-442$

$-479$

$-2.77$

0.02

$-7.12$

$-356$

$-494$

$-28.01$

$-393$

$-5.13-0.50 \quad-28.01$

$-1.43$

2.82

25,790

440

$-6.52$

1.07

262.06

$-1.47 \quad-0.20$

$-728$

$\begin{array}{llll}-6.69 & -0.08 & -44.72 & -\end{array}$

$-696$

$\begin{array}{rcccccccc}\text { in levels } & -2.12 & -2.29 & 556 & 1092 & -2.56 & -3.52 & 543 & 768 \\ \text { in } \operatorname{logs}(\times 100) & -9.93 & -0.89 & 23.97 & - & -11.96 & -1.36 & 23.78 & -\end{array}$

\footnotetext{
† Notes: Entries in the Table represent changes (in levels and in logs) in the outcomes as a result of a given policy. The unrestricted model parameters are the maximum likelihood estimates in the medium wage sample. The restricted model parameters are the maximum likelihood estimates in the medium wage sample subject to the restriction that $\beta=1$. Source: Author's calculations.
} 


\section{$\underline{\text { Appendix Table 1: Methods of Search }}{ }^{\dagger}$}

Summary Statistics

(1) (2)
(3)

(4)
Maximum likelihood estimates

(5)

(6)

\begin{tabular}{|c|c|c|c|c|c|c|}
\hline $\begin{array}{c}\text { Method of } \\
\text { Search }\end{array}$ & $\begin{array}{l}\text { Percentag } \\
\text { e using } \\
\text { method } \\
\end{array}$ & $\begin{array}{l}\text { Average hours } \\
\text { conditional on } \\
\text { using method }\end{array}$ & $\begin{array}{l}\text { Monthly } \\
\text { probability of } \\
\text { receiving offer }\end{array}$ & $\begin{array}{l}\text { Implied weekly } \\
\text { probability of } \\
\text { receiving offer }\end{array}$ & $\begin{array}{c}\text { Estimate } \\
\text { of } \alpha\end{array}$ & $\begin{array}{c}\text { Estimate } \\
\text { of } \mathrm{c}\end{array}$ \\
\hline $\begin{array}{l}\text { State } \\
\text { Employment } \\
\text { Service }\end{array}$ & 51.66 & 3.54 & 13.37 & 3.52 & $\begin{array}{c}0.0309 \\
(0.0062)\end{array}$ & $\begin{array}{c}0.7183 \\
(0.1864)\end{array}$ \\
\hline $\begin{array}{l}\text { Friends and } \\
\text { Relatives }\end{array}$ & 70.63 & 3.61 & 15.57 & 4.14 & $\begin{array}{c}0.0387 \\
(0.0045)\end{array}$ & $\begin{array}{c}0.8874 \\
(0.1796)\end{array}$ \\
\hline $\begin{array}{l}\text { Newspaper } \\
\text { ads }\end{array}$ & 65.54 & 4.01 & 9.28 & 2.41 & $\begin{array}{c}0.0200 \\
(0.0038)\end{array}$ & $\begin{array}{c}0.4209 \\
(0.1067)\end{array}$ \\
\hline $\begin{array}{l}\text { Direct } \\
\text { employer } \\
\text { contact }\end{array}$ & 62.72 & 4.77 & 17.46 & 4.68 & $\begin{array}{c}0.0358 \\
(0.0046)\end{array}$ & $\begin{array}{c}0.6566 \\
(0.1368)\end{array}$ \\
\hline $\begin{array}{l}\text { Other } \\
\text { methods }\end{array}$ & 56.32 & 3.33 & 17.77 & 4.77 & $\begin{array}{c}0.0409 \\
(0.0068)\end{array}$ & $\begin{array}{l}1^{*} \\
(-)\end{array}$ \\
\hline All Methods & 100.00 & 7.17 & 32.93 & 9.50 & - & - \\
\hline
\end{tabular}

\footnotetext{
† Notes: Summary statistics based on author's calculations using the 1981 Job Search questionnaire in the NLSY. Maximum likelihood estimates of $\alpha$ and $c$ based on the model described in the Appendix.

* The cost coefficients are identified only up to a scale factor. The cost coefficient for "Other Methods" is therefore fixed at 1.
} 
Model Dynamics: The Probability of Exiting Unemployment

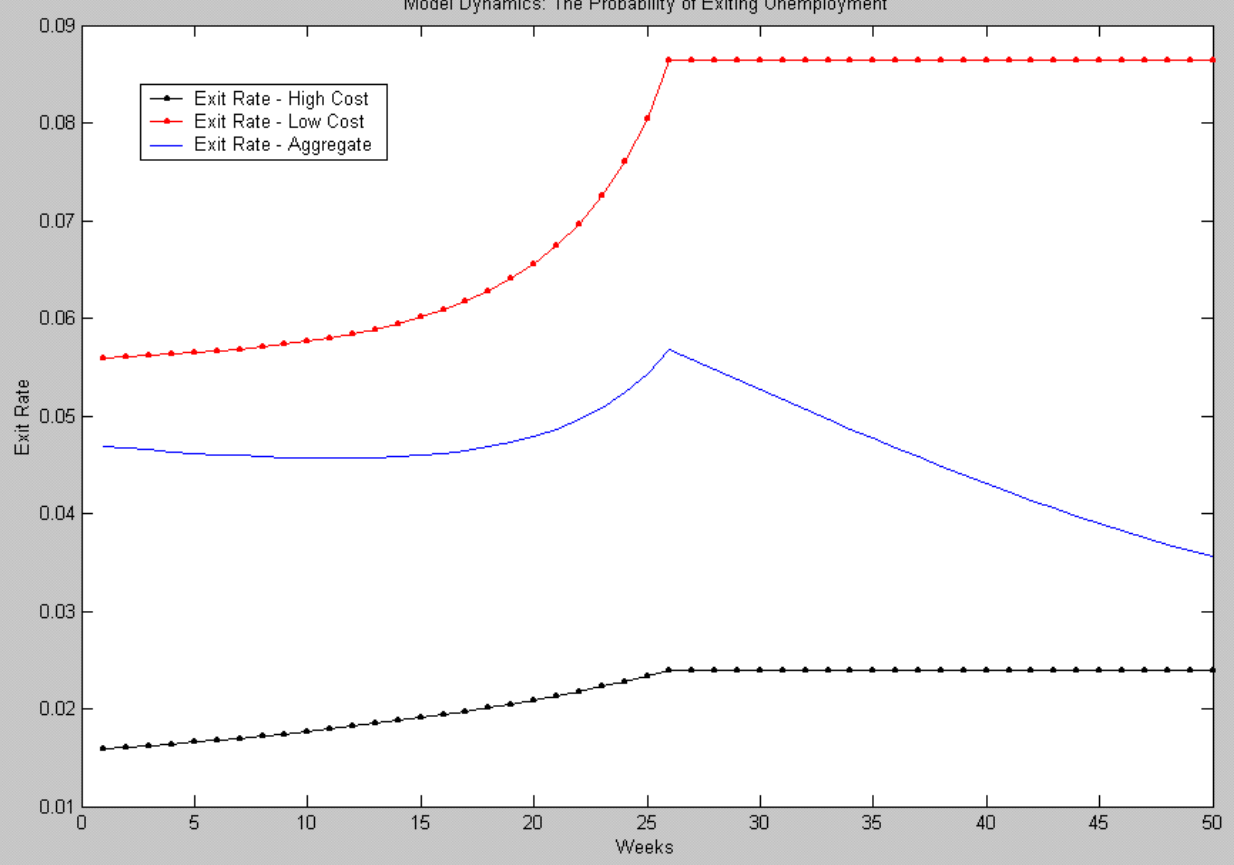

Model Dynamics: Expected Re-employment Wage

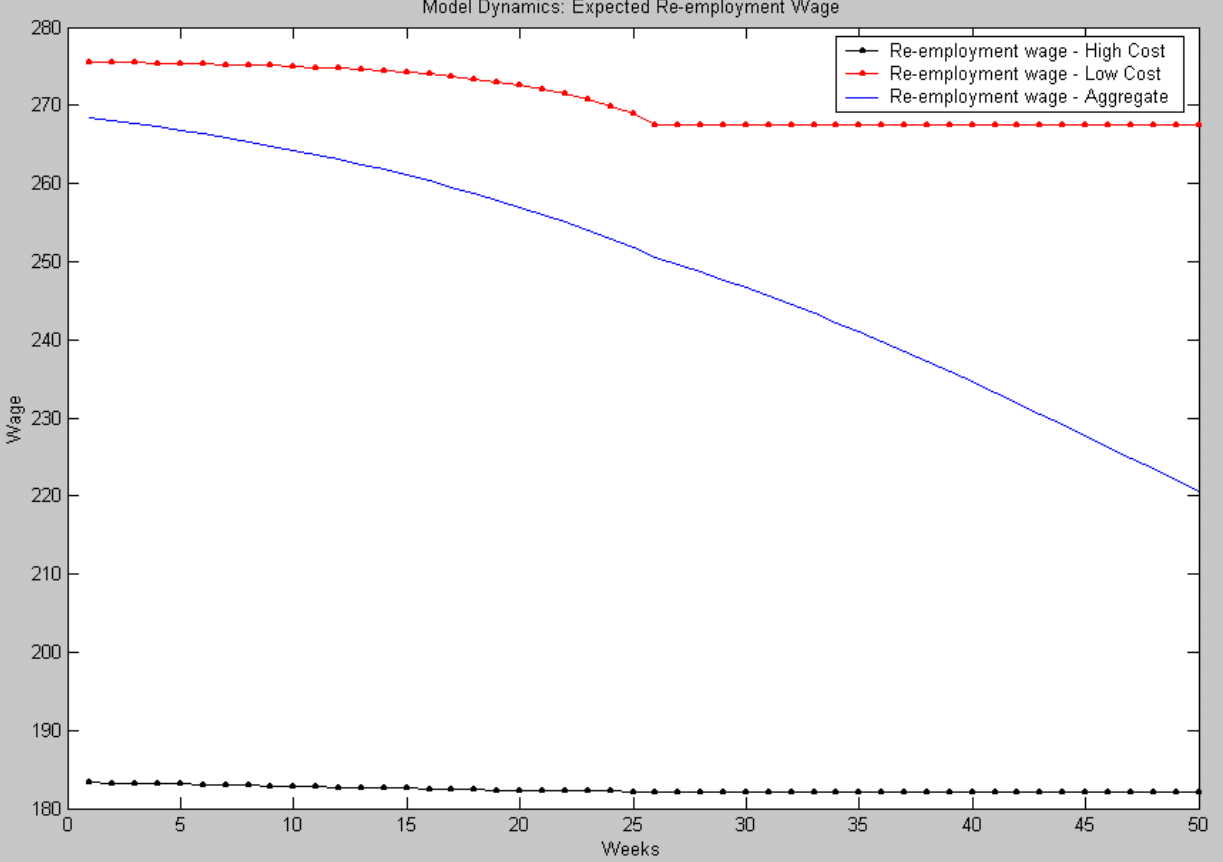

Figure 1: Model Dynamics 

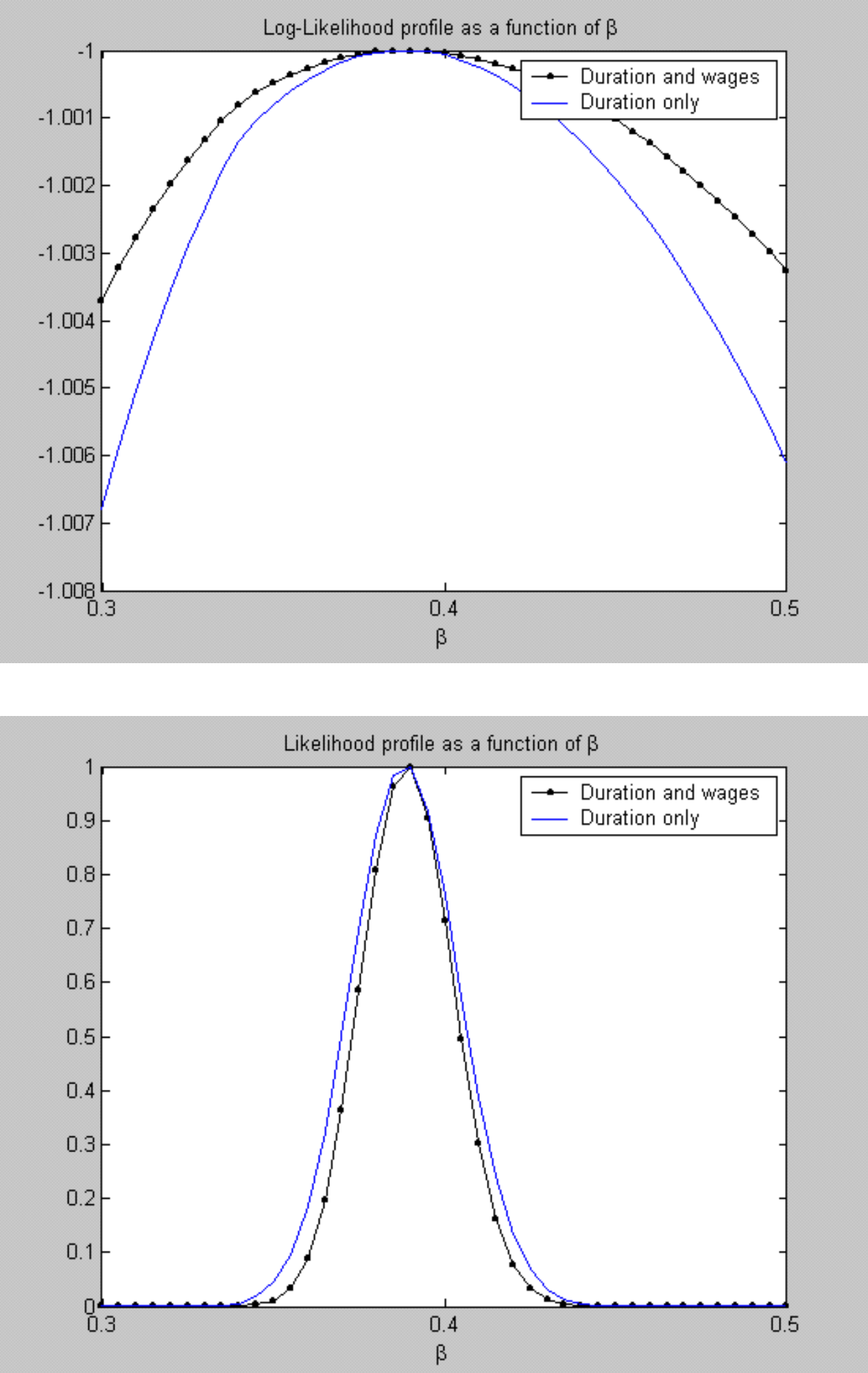

Figure 2a: Likelihood Profiles as a Function of $\beta$ 

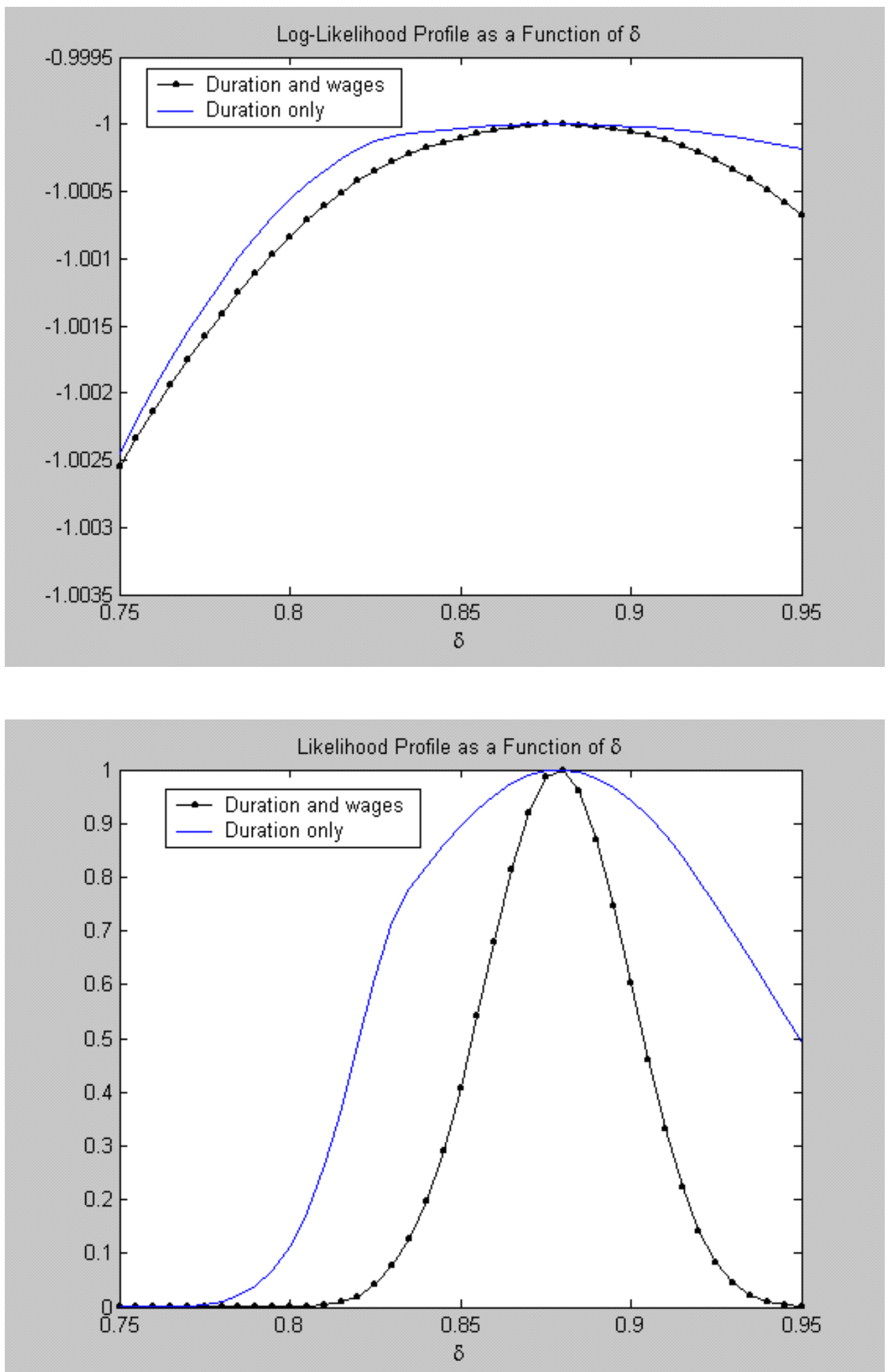

Figure 2b: Likelihood Profiles as a Function of $\delta$ 

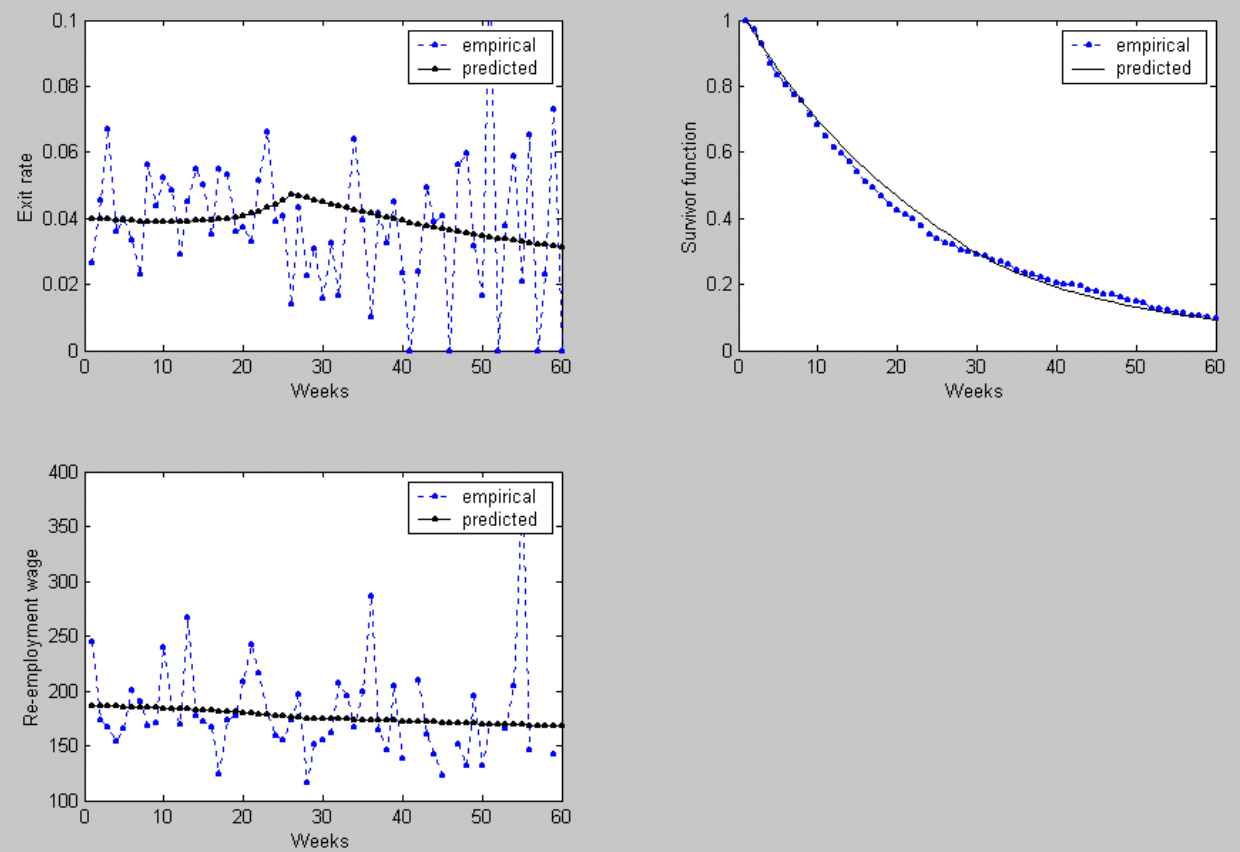

Figure 3a: Job Search Dynamics, Low Wage Sample 

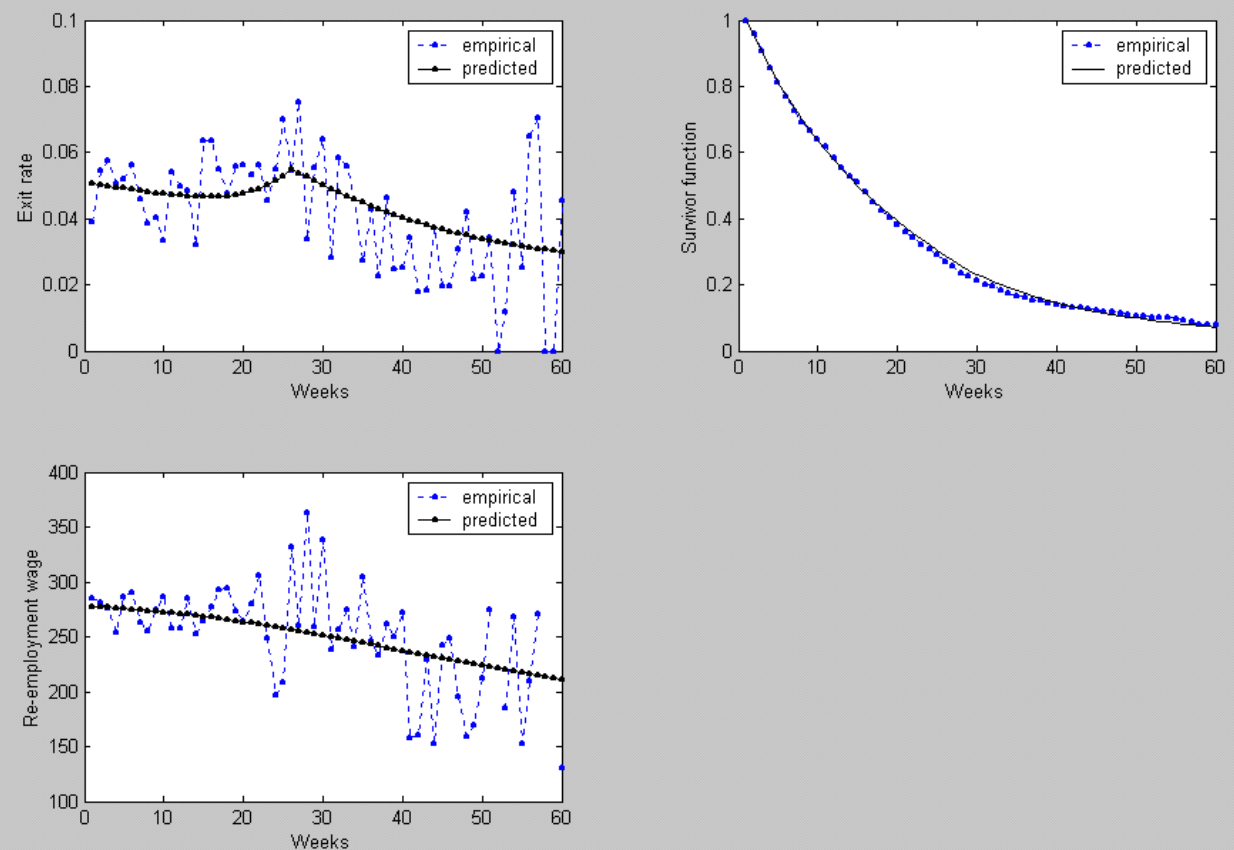

Figure 3b: Job Search Dynamics, Medium Wage Sample 

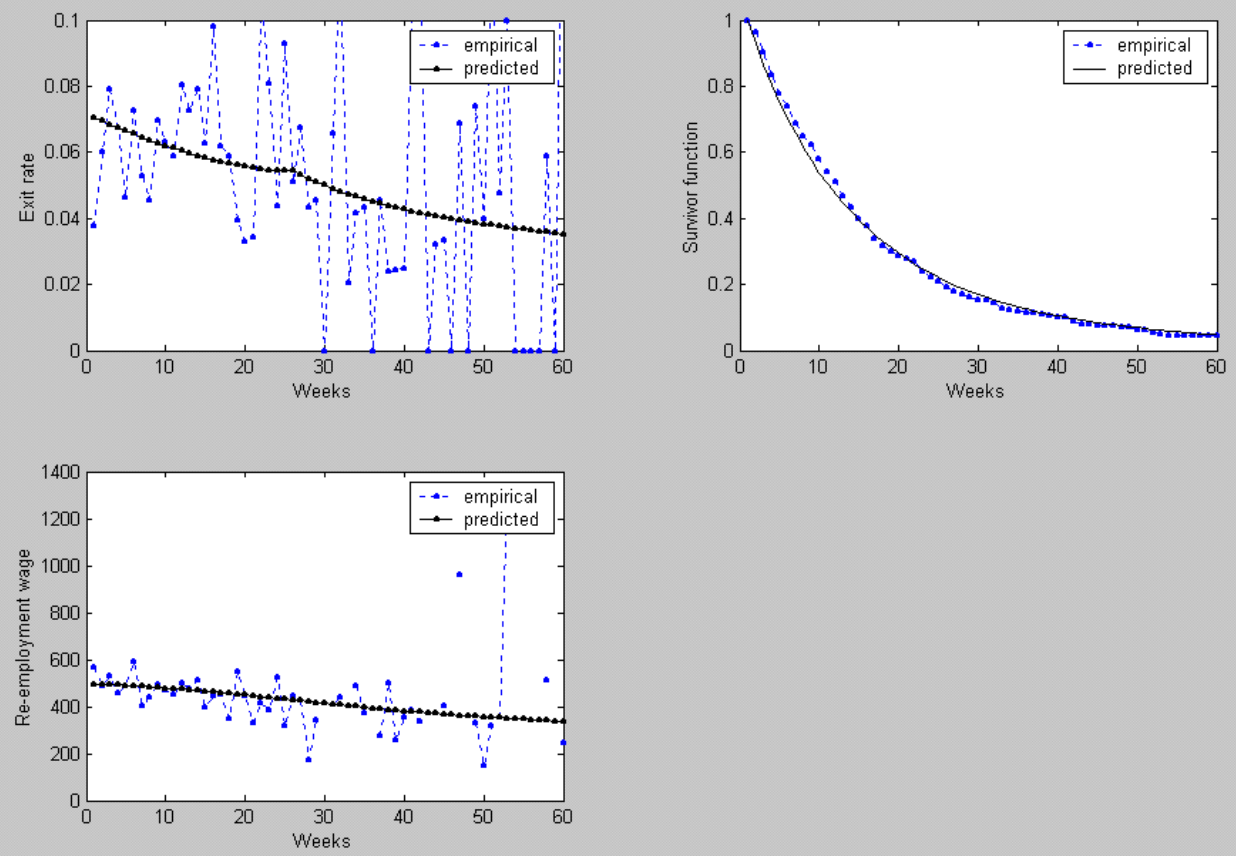

Figure 3c: Job Search Dynamics, High Wage Sample 

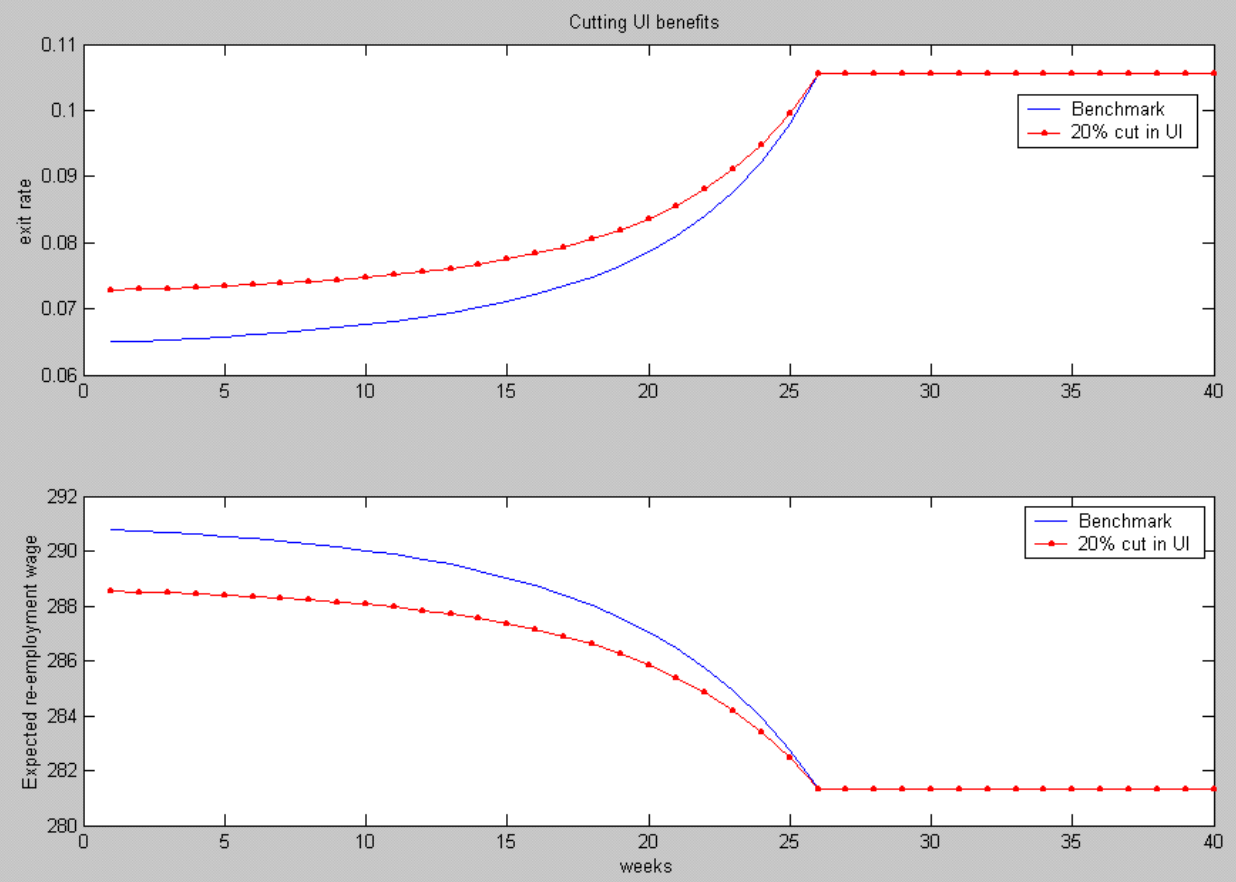

Figure 4a: Cutting UI Benefits
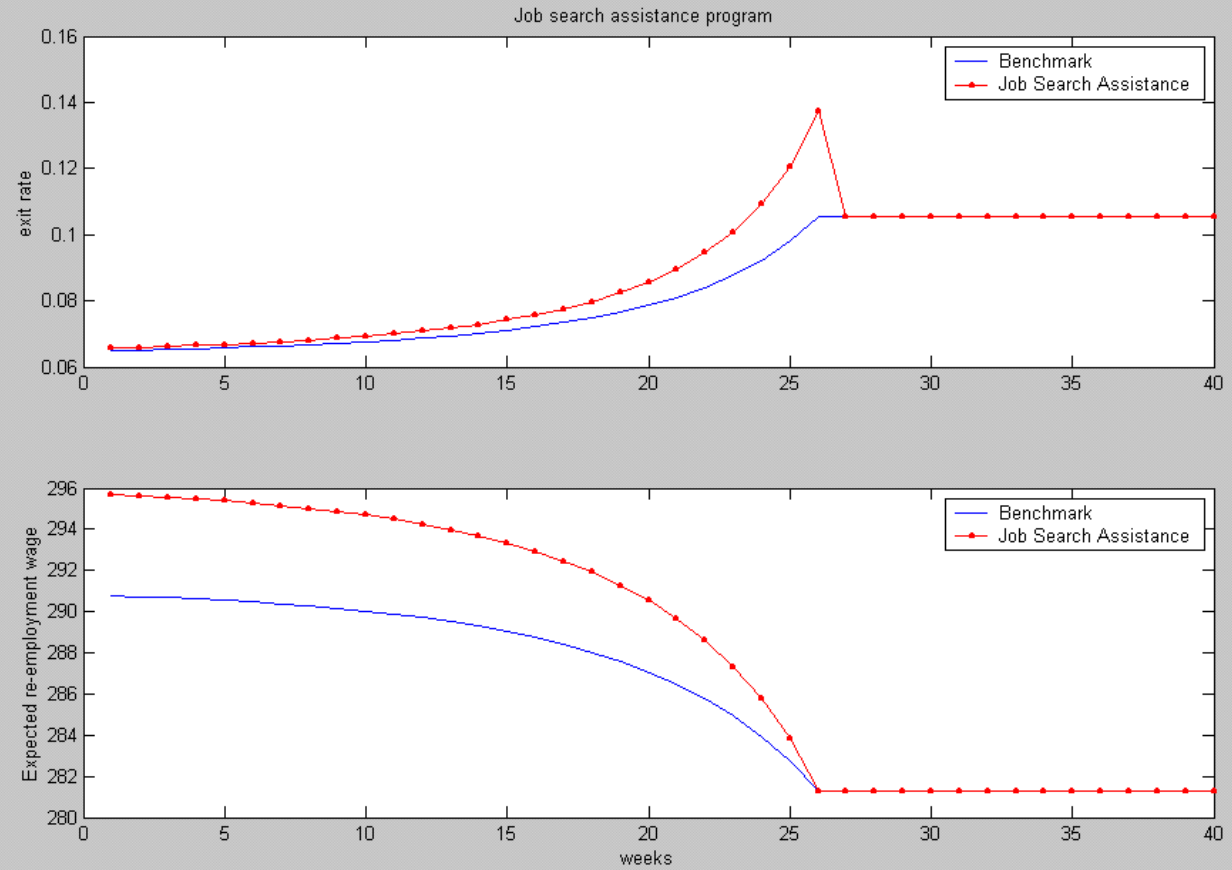

Figure 4b: Job Search Assistance Program 

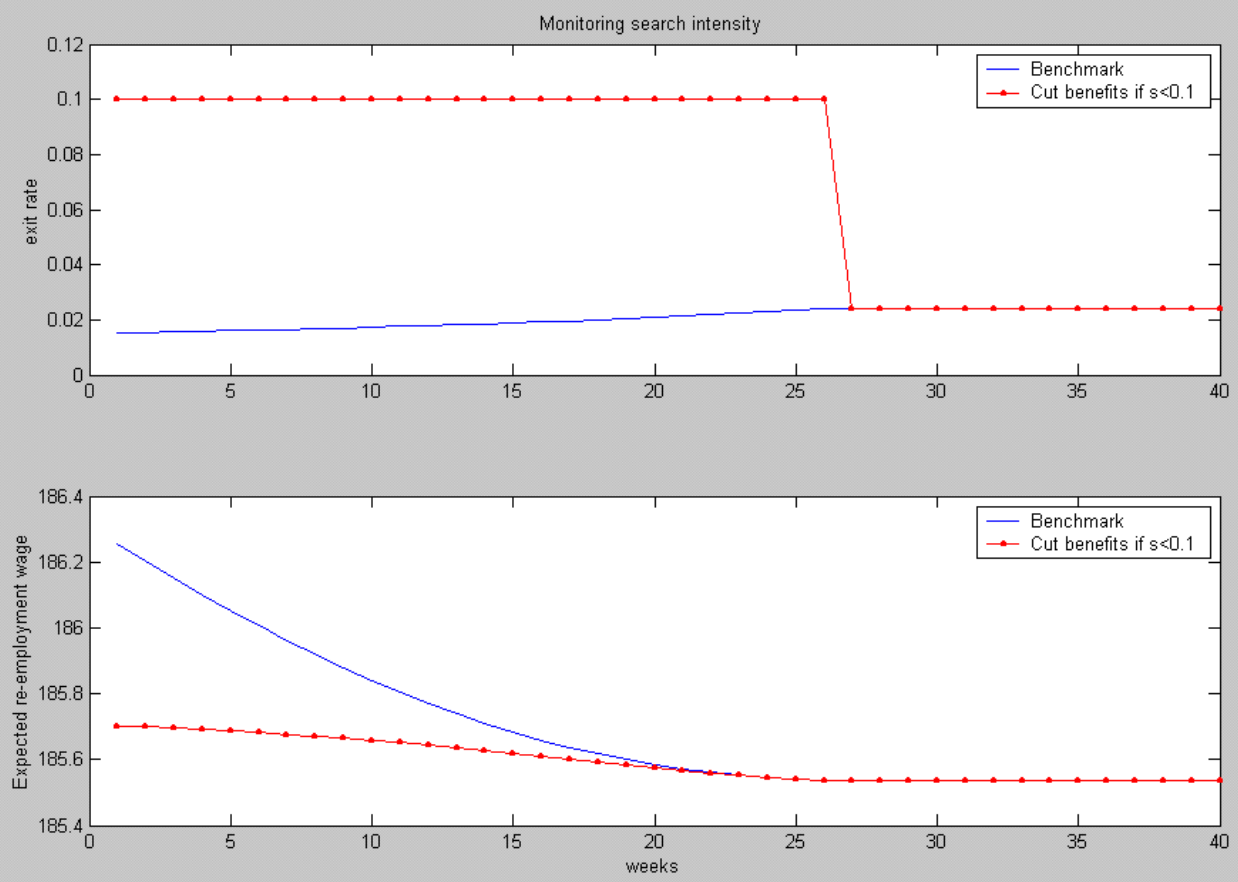

Figure 4c: Monitoring Search Intensity
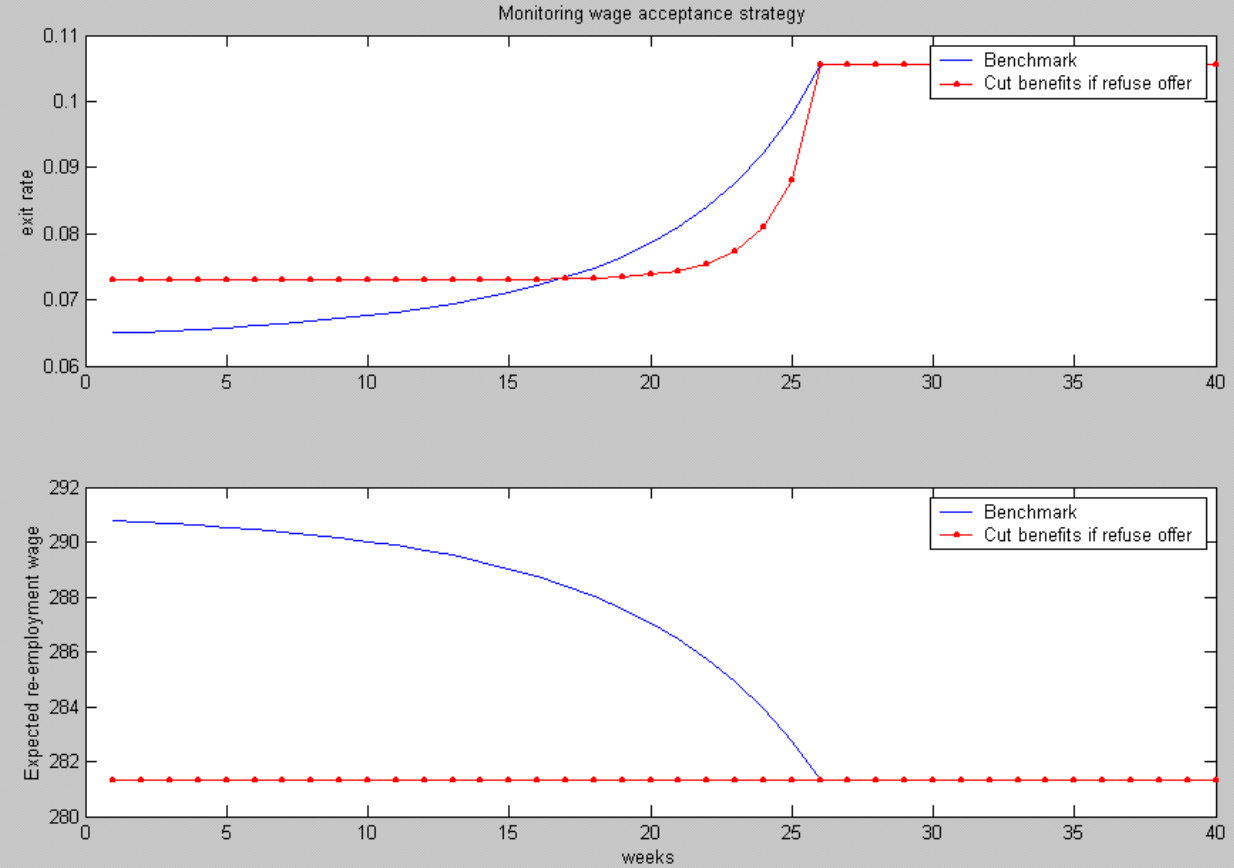

Figure 4d: Monitoring the Wage Acceptance Strategy 

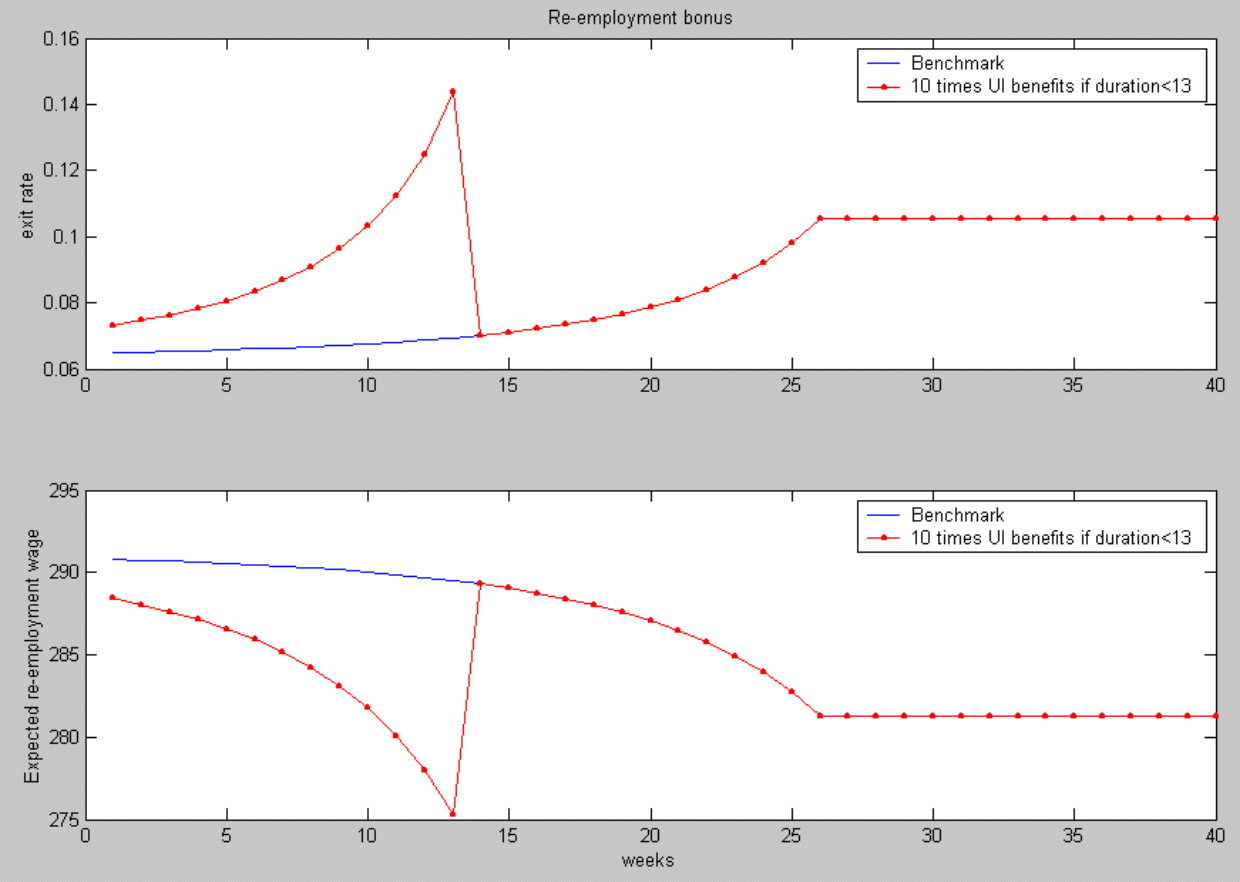

Figure 4e: Re-employment Bonus 


\section{IZA Discussion Papers}

\begin{tabular}{|c|c|c|c|c|}
\hline No. & Author(s) & Title & Area & Date \\
\hline 983 & $\begin{array}{l}\text { D. Del Boca } \\
\text { M. Locatelli } \\
\text { D. Vuri }\end{array}$ & Child Care Choices by Italian Households & 3 & $01 / 04$ \\
\hline 984 & $\begin{array}{l}\text { W. Arulampalam } \\
\text { A. L. Booth } \\
\text { M. L. Bryan }\end{array}$ & $\begin{array}{l}\text { Are there Asymmetries in the Effects of Training } \\
\text { on the Conditional Male Wage Distribution? }\end{array}$ & 5 & $01 / 04$ \\
\hline 985 & $\begin{array}{l}\text { Š. Jurajda } \\
\text { H. Harmgart }\end{array}$ & When Are 'Female' Occupations Paying More? & 4 & $01 / 04$ \\
\hline 986 & $\begin{array}{l}\text { H. Brücker } \\
\text { P. Trübswetter }\end{array}$ & $\begin{array}{l}\text { Do the Best Go West? An Analysis of the Self- } \\
\text { Selection of Employed East-West Migrants in } \\
\text { Germany }\end{array}$ & 1 & $01 / 04$ \\
\hline 987 & $\begin{array}{l}\text { A. Ichino } \\
\text { G. Muehlheusser }\end{array}$ & $\begin{array}{l}\text { How Often Should You Open the Door? Optimal } \\
\text { Monitoring to Screen Heterogeneous Agents }\end{array}$ & 7 & $01 / 04$ \\
\hline 988 & M. Jansen & Can Job Competition Prevent Hold-Ups? & 7 & $01 / 04$ \\
\hline 989 & J. Wagner & $\begin{array}{l}\text { Are Young and Small Firms Hothouses for } \\
\text { Nascent Entrepreneurs? Evidence from German } \\
\text { Micro Data }\end{array}$ & 1 & $01 / 04$ \\
\hline 990 & $\begin{array}{l}\text { H. Bonin } \\
\text { C. Patxot }\end{array}$ & $\begin{array}{l}\text { Generational Accounting as a Tool to Assess } \\
\text { Fiscal Sustainability: An Overview of the } \\
\text { Methodology }\end{array}$ & 7 & $01 / 04$ \\
\hline 991 & S. Verick & $\begin{array}{l}\text { Threshold Effects of Dismissal Protection } \\
\text { Legislation in Germany }\end{array}$ & 1 & $01 / 04$ \\
\hline 992 & A. Heitmueller & $\begin{array}{l}\text { Public-Private Sector Wage Differentials in } \\
\text { Scotland: An Endogenous Switching Model }\end{array}$ & 7 & $01 / 04$ \\
\hline 993 & $\begin{array}{l}\text { A. Calderon-Madrid } \\
\text { A. Voicu }\end{array}$ & $\begin{array}{l}\text { Total Factor Productivity Growth and Job } \\
\text { Turnover in Mexican Manufacturing Plants in the } \\
\text { 1990s }\end{array}$ & 4 & $01 / 04$ \\
\hline 994 & $\begin{array}{l}\text { M. Fertig } \\
\text { R. E. Wright }\end{array}$ & $\begin{array}{l}\text { School Quality, Educational Attainment and } \\
\text { Aggregation Bias }\end{array}$ & 1 & $01 / 04$ \\
\hline 995 & $\begin{array}{l}\text { G. Brunello } \\
\text { M. Giannini } \\
\text { K. Ariga }\end{array}$ & The Optimal Timing of School Tracking & 5 & $01 / 04$ \\
\hline 996 & M. D. Paserman & $\begin{array}{l}\text { Bayesian Inference for Duration Data with } \\
\text { Unobserved and Unknown Heterogeneity: } \\
\text { Monte Carlo Evidence and an Application }\end{array}$ & 7 & $01 / 04$ \\
\hline 997 & M. D. Paserman & $\begin{array}{l}\text { Job Search and Hyperbolic Discounting: } \\
\text { Structural Estimation and Policy Evaluation }\end{array}$ & 6 & $01 / 04$ \\
\hline
\end{tabular}

An updated list of IZA Discussion Papers is available on the center's homepage www.iza.org. 